Article

\title{
The Net Worth Trap: Investment and Output Dynamics in the Presence of Financing Constraints
}

\author{
Jukka Isohätälä ${ }^{1}$, Alistair Milne ${ }^{2, *} \mathbb{1}$ and Donald Robertson ${ }^{3}$ \\ 1 Department of Operational Research, National University of Singapore, Singapore 119077, Singapore; \\ jukka.isohatala@gmail.com \\ 2 School of Business and Economics, Loughborough University, Epinal Way, Loughborough LE11 3TU, UK \\ 3 Faculty of Economics, University of Cambridge, Cambridge CB3 9DD, UK; dr10011@cam.ac.uk \\ * Correspondence: a.k.l.milne@lboro.ac.uk
}

Received: 13 July 2020; Accepted: 7 August 2020; Published: 10 August 2020

\begin{abstract}
This paper investigates investment and output dynamics in a simple continuous time setting, showing that financing constraints substantially alter the relationship between net worth and the decisions of an optimizing firm. In the absence of financing constraints, net worth is irrelevant (the 1958 Modigliani-Miller irrelevance proposition applies). When incorporating financing constraints, a decline in net worth leads to the firm reducing investment and also output (when this reduces risk exposure). This negative relationship between net worth and investment has already been examined in the literature. The contribution here is providing new intuitive insights: (i) showing how large and long lasting the resulting non-linearity of firm behaviour can be, even with linear production and preferences; and (ii) highlighting the economic mechanisms involved-the emergence of shadow prices creating both corporate prudential saving and induced risk aversion. The emergence of such pronounced non-linearity, even with linear production and preference functions, suggests that financing constraints can have a major impact on investment and output; and this should be allowed for in empirical modelling of economic and financial crises (for example, the great depression of the 1930s, the global financial crisis of 2007-2008 and the crash following the Covid-19 pandemic of 2020).
\end{abstract}

Keywords: cash flow management; corporate prudential risk; the financial accelerator; financial distress; induced risk aversion; liquidity constraints; liquidity risk; macroeconomic propagation; multiperiod financial management; non-linear macroeconomic modelling; Tobin's q; precautionary savings

\section{JEL Classification: E44}

\section{Introduction}

This paper examines the impacts of financing constraints on firm operations and finances using the tools of continuous time dynamic stochastic optimisation. The introduction of a threshold whereat the firm faces costly refinancing or liquidation, changes the behaviour of a firm, even when the firm's shareholders are risk neutral. As the threshold is approached, there emerges an increasing premium on the value of cash held inside the firm (relative to the outside cost of capital) and an increasing aversion to risk.

This modelling builds on the dynamic analyses of [1,2]. Following [2] firms have constant returns to scale, i.e., linear production technology, and seek to maximise the value of cash dividends paid to share- holders whilst facing convex costs of adjusting their capital stock. With these assumptions the solution to the firm's optimisation problem can be expressed in terms of a single state variable, the ratio 
of balance sheet net worth to productive capital. Cash held internally reduces shareholder returns but also lowers the expected future costs of refinancing or liquidation. It is the interplay between these two forces that drives behaviour.

The financing constraint is assumed to be an exogenous (to the firm) lower boundary for this state variable at which the firm must undertake costly refinancing or bankruptcy occurs and the firm is liquidated. An upper bound appears as part of the optimal solution and marks the threshold where the firm pays out cash flow dividends to its impatient shareholders. As shocks drive the firm closer to the liquidation threshold, its presence increasingly affects the optimal decisions of the firm.

An analogy for this mechanism is provided by Whittle [3] pages 287-288:

This might be termed the "fly-paper" effect... A deterministic fly, whose path is fully under its own control, can approach arbitrarily closely to the fly-paper with impunity, knowing he can avoid entrapment ... A stochastic fly cannot guarantee his escape; the nearer he is to the paper, the more certain it is that he will be carried onto it. This also explains why the fly tries so much harder in the stochastic case than in the deterministic case to escape the neighbourhood of the fly-paper... One may say that the penalty of ending on the fly-paper "propagates" into the free-flight region in the stochastic case, causing the fly to take avoiding action while still at a distance from the paper.

Whittle is pointing out that in dynamic settings with (i) uncertainty in the equations of motion for state variables (the position of the fly); and (ii) constraints on state (the fly paper), then (in the language of economic theory) non-zero shadow prices appear even for values of the state where the constraints are not currently binding.

In the setting of this paper there are two routes by which this mechanism affects firm decisions:

(1) A shadow price of internal funds creates a wedge between the internal and external cost of capital. This reduces the marginal valuation of investment in terms of internal funds (Tobin's marginal-q). A firm invests less and less as its net worth declines. The consequence is corporate prudential saving, analogous to the household prudential saving extensively discussed in the literature on the consumption function (surveyed in [4]).

(2) A shadow price of risk creates an "induced risk aversion" leading to firms reducing their risk exposure. A variation of the model allows firms to respond by renting out more and more of their capital as net worth declines below a threshold level. It is this mechanism which, if sufficiently powerful, creates the "net worth trap."

Since the firm cannot raise new equity capital, declines in net worth are financed by increases in firm borrowing. When this state variable is comparatively high, near the upper boundary where dividends are paid, then these shadow prices are close to those that would apply for a financially unconstrained firm that can raise new equity. As the state variable falls closer to its minimum level at which no further borrowing is possible, these shadow prices rise. Even with the assumed linear production technology and the risk-neutrality of firm shareholders, the resulting dynamics of corporate output and investment are highly non-linear, depending on both the direction and size of shocks: negative shocks to productivity or net worth have a larger impact than positive shocks; small negative shocks self-correct relatively quickly; larger negative shocks (or a succession of smaller negative shocks) can result in extended periods of high shadow prices and contractions of output and investment.

The remainder of the paper is set out as follows. Section 2 locates the paper in the economics, finance and mathematical insurance literature. Section 3 presents a simplified version of the model in which capital cannot be rented out. For high values of the fixed cost of recapitalisation, firms do not recapitalise instead liquidating on the lower net worth boundary; but for lower values firms choose to exercise their option to recapitalise on the lower boundary and so avoid liquidation. In either case, investment is reduced below unconstrained levels by the state dependent shadow price of internal funds. 
Section 4 then introduces the possibility that firms, by mothballing or renting capital to outsiders, are able to reduce their risk exposure, but at the expense of a decline in their expected output. The extent to which this is done depends on the magnitude of a shadow price of risk capturing an effective induced risk aversion for other wise risk-neutral shareholders. This is where the possibility of a net worth trap emerges. Section 5 provides a concluding discussion considering the macroeconomic implications of these findings. While the model solution is numerical, not closed form, we have developed convenient and rapid solution routines in Mathematica. (We have created a standalone module which can be used by any interested reader to explore the impact of parameter choice on model outcomes. This, together with the Mathematica notebook used for creating the Figures reported in the paper, can be downloaded via http:/ / leveragecycles.lboro.ac.uk/networthtrap.html and run using the free Mathematica Player software https://www.wolfram.com/player/). Four appendices contain supporting technical details. (Appendix A solves the situation in which there is no non-negativity constraint on dividend payments; or equivalently when uncertainty vanishes. Appendix B provides proofs of the propositions in the main text. Appendix D derives the asymptotic approximations used to incorporate the singularities that arise in the model with rent. Appendix $C$ details the numerical solution, noting how this must be handled differently in the two possible cases, wherein a "no Ponzi" condition applies to the unconstrained model of Appendix A; and when this condition does not).

\section{Related Literature}

There is a substantial body of literature examining firm operations, financing and risk management over multiple periods. Central to this work is the inventory theoretic modelling (initiated by $[5,6]$ )) of both financial (cash, liquidity and capitalisation) and operational (inventory, employment, fixed capital investment) decisions subject to fixed (and sometimes also proportional or convex) costs of replenishment or investment.

Most dynamic models of corporate behaviour focus either on financial or operational decisions without considering their interaction. Well known contributions include work on the dynamics of fixed capital investment in the presence of adjustment costs (including $[7,8]$ ); and on applying standard tools of inventory modelling to study corporate cash holdings and money demand [9-11]. Dynamic modelling methods are also employed in the contingent claims literature, to examine both the pricing of corporate liabilities [12] and the possibility of strategic debt repudiation [13,14] and the interaction of the choice of asset risk and capital structure, taking account of the implications for the cost of debt [15]. However, this line of research does not address the dynamic interaction of financing and investment.

The interaction of financial and operational decisions is often considered in a static framework. This allows an explicit statement of the informational asymmetries and strategic interactions that lead to departures from the [16] irrelevance proposition (for a unified presentation of much of this literature, see [17]). This is widely used in the corporate finance literature. Take, for example, the pecking order theory of capital structure in which costs of equity issuance result in discrepancies between the costs of inside funds (retained earnings), debt and outside equity [18,19]. In [20] such a static framework was applied to develop a joint framework of the determination of investment and risk management decisions.

There is a smaller body of literature on the dynamic interactions of financial and operational decisions. The negative relationship between net worth and the shadow price of internal funds that appears in the present paper is not a new finding; it appears in a number of other contributions to the literature. In [21] the costly state verification problem of [22] is extended into a recursive model of dynamic stochastic control wherein one period debt contract can be refinanced through a new debt contract. His analysis does not establish an explicit solution for the optimal contract, but it does show how if debt contracts are used to dynamically finance a productive investment opportunity, then the value function has a "characteristic" convex shape, with a negative second derivative with respect to net worth, reflecting a departure from [16] capital structure irrelevance and a resulting shadow price of internal funds. In consequence, as net worth declines so does investment and output. 
Progress has been made more recently on analysing optimal financial contracts in a dynamic principal agent context (see $[23,24]$ and references therein), yielding similar divergence between the cost of funds. In [24] it is shown that it can be optimal for a firm to use, simultaneously, both long term debt and short term lines of credit, in order to create incentives for managerial effort, but this work has not been extended to modelling the interactions of financial and operational decisions.

Most other work on the dynamic interactions of financial and operational decisions has proceeded, as in this paper, by imposing costly financial frictions (rather than establishing an optimal contract). Many of these papers employ continuous time modelling techniques. An early example is [25] exploring the bond financing of a project subject to fixed costs both of opening and shutting the project (hence creating real option values) and of altering capital structure through bond issuing. Four papers written independently [1,26-28] explore cash flow management and dividend policy in a context where cash holdings evolve stochastically (as a continuous time diffusion) resulting in a need for liquidity management. This leads to the simple boundary control for dividends that is inherited by the model of the present paper: paying no dividends when net cash holdings are below a target level and making unlimited dividend payments on this boundary.

This cash flow management framework was subsequently employed in a variety of different contexts. These include the risk exposure decisions of both insurance companies and non-financial corporates (see [29-31]). In a sequence of papers [32-35] bank capital regulation and bank behaviour are analysed. Other related work examined how intervention rules affect market pricing in exchange rates and in money markets; for example, [36-38] provides a survey article linking this work to portfolio allocation and cash management problems faced by companies and insurance firms.

Other recent and closely related studies exploring the interactions of financing, risk management and operational decisions include [39-44]. While employing differing assumptions, these papers have a great deal in common. The resulting dynamic optimisation again yielded a value function with the "characteristic" convex shape reported by [21] and appearing also in this paper, and hence resulted in internal "shadow prices" which reduce risk exposure, output and investment as net worth or cash holdings decline.

Similar findings emerged in discrete time models, such as those of $[45,46]$, who considered risk management and firm decision making in the presence of taxation and imposed costs of financial transactions. They incorporated a wide range of determining factors and firm decision variables, again finding that a reduction in net worth leads to reduced of risk exposure and increased incentives to hedge risks.

While the literature offers a consistent account of the dynamic interactions of corporate financing and operational decisions, the macroeconomic implications are less fully explored. Capital market frictions, in particular the high costs of external equity finance and the role of collateral values have been proposed as an explanation of macroeconomic dynamics (see, e.g., $[47,48])$. The most widely used implementation of these ideas is the "financial accelerator" introduced into macroeconomics by $[49,50]$. This is based on a static model of underlying capital market frictions, in which the macroeconomic impact of financing constraints comes through assuming a costly state verification (as in [22]) and modelling the resulting difficulties entrepreneurs face in obtaining external finance for new investment projects. The resulting propagation mechanism operates through an "external financing premium," i.e., a additional cost that must be paid by investors in fixed capital projects in order to overcome the frictional costs of external monitoring whenever they raise external funds, rather than internal shadow prices such as appear in this paper.

An alternative perspective on the propagation of macroeconomic shocks is found in the literature on endogenous risk in traded asset markets (see [51-53] in which asset price volatility limits access to external finance. In their overview of the impacts of financing constraints on macrofinancial dynamics using continuous-time modelling [54] highlighted some further recent analysis of this kind [2,55,56] that focused on the impacts of financial constraints on asset prices. 
In $[55,56]$ "specialists" were able to better manage financial assets but their ability to invest in these assets was constrained by their net worth. The outcome was two regimes: one of higher net worth wherein the constraint does not bind and the pricing and volatility of assets are determined by fundamental future cash flows; the other of lower net worth wherein asset prices fall and asset price volatility rises relative to fundamental levels. This approach is developed further in [2], treating physical capital as a tradable financial asset and showing how endogenous volatility can then limit investment and create the possibility of a "net worth" trap with extended declines in output and investment following a major negative shock.

The analysis of this paper shows that such a net worth trap can also emerges through the operation of internal shadow prices, rather than, as in [2], through external market pricing. Thus there is a potential net worth trap for all firms, large and small and regardless of the extent to which they participate in external financial and asset markets. This paper also extends [2] by allowing not just for shocks to capital productivity (these can be interpreted as supply shocks such as those that have resulted from the Covid-19 pandemic) but also for shocks to net worth (these can be interpreted as demand and financial market shocks, such as those that occurred during the global financial crisis). As in [2], in order to limit the number of state variables and obtain tractable results, this paper considers only serially uncorrelated shocks represented in continuous time by a Wiener process.

This paper contributes to this literature in two further ways. First, building on [1] it distinguishes the impact on firm decisions of the shadow price of internal funds (the first derivative of the value function in net worth) from the shadow price of risk (the scaled second derivative of the value function). Second, it develops efficient numerical solution methods which support convenient exploration of the effect of changing parameters on firm decisions.

\section{A Basic Model}

This section solves a basic model in which firms decide only on investment and dividend payments. We delay to Section 4 consideration of a broader model in which firms can reduce their risk exposure by selling or renting capital to outsiders, and the circumstances in which a net worth trap might then appear.

Section 3.1 sets out the model assumptions. Section 3.2 discusses its numerical solution. Section 3.3 presents some illustrative simulations of the numerical solution.

\subsection{Model Assumptions}

Firms produce output sold at price unity through a constant returns to scale production function $(y=a k)$ and seek to maximise a flow of cash dividends $(\lambda \geq 0)$ to risk neutral owners/shareholders. There are two state variables, capital $k$, which is augmented by investment at rate $i$ and reduced by depreciation at rate $\delta$, and cash, which increases with sale of output and is reduced by capital investment (subject to quadratic adjustment costs) and dividend payouts. Cash $c$ held internally (which can be negative if borrowing) attracts an interest rate $r$. Additionally, the firm can recapitalise (increase its cash) at any time $\tau$ by an amount $\epsilon(\tau)$ where $\tau$ can be chosen by the firm. Cash holdings are disturbed by an amount $\sigma k d z$ with $d z$ a Wiener process.

The state variables evolve according to:

$$
\begin{aligned}
& d c=\left[-\lambda+a k+r c-i k-\frac{1}{2} \theta(i-\delta)^{2} k\right] d t+\epsilon(\tau)+\sigma k d z \\
& d k=(i-\delta) k d t
\end{aligned}
$$

where the coefficient $\theta$ captures costs of adjustment of the capital stock (increasing with the net rate of investment $i-\delta$ ). 
The firm seeks to maximise the objective:

$$
\Omega=\max _{\left\{i_{t}\right\},\left\{\lambda_{t}\right\},\left\{\epsilon_{\tau}\right\}} E \int_{t=0}^{\infty} e^{-\rho t} \lambda d t-\sum_{\tau=\tau_{1}}^{\tau_{\infty}} e^{-\rho \tau}\left(\epsilon_{\tau}+\chi k\right)
$$

where $\chi>0$ represents the cost to shareholders of recapitalisation, arising from any associated due-diligence or dilution of interests. These are assumed proportional to $k$.

The only other agents are outside investors ("households" in the terminology of [2] who lend to firms, but do not take credit risks; instead, they require that lending is secured against the firm's assets, limiting the amount of credit available to the firm, and they become the residual owner of the firm's assets if and when the debt is not serviced. Like firm owners, these investors are risk-neutral and seek to maximise the present discounted value of current and future consumption. Unlike firms, there is no non-negativity constraint on their cash flow. Since they are the marginal suppliers of finance, and there is no risk of credit losses, they lend to or borrow from firms at a rate of interest $r$ reflecting their rate of time discount. Investors are more patient than firms, i.e., $r<\rho$ (without this assumption firms will build up unlimited cash holdings instead of paying dividends). Fixed capital held directly by outside investors generates an output of $\bar{a} k$.

Further assumptions are required in order to obtain a meaningful solution: (i) capital is less productive in the hands of outside investors than when held by firms (otherwise, firms will avoid using capital for production), $\bar{a}<a$; (ii) upper bounds on both $a$ and $\bar{a}$ to ensure that the technology does not generate sufficient output to allow self-sustaining growth faster than the rates of shareholder or household discount; (iii) a further technical condition (a tighter upper bound on $a$ ) ensuring that there is a solution in which dividends are paid to firm shareholders.

\subsection{Solution}

\subsubsection{Characterisation of the Solution}

The form of the solution is summarised in the following propositions:

Proposition 1. The firm can borrow (hold $c<0$ ) up to a maximum amount determined by the valuation of the firm by outside investors:

$$
c>\bar{\eta} k=-\left[1+\theta\left(r-\sqrt{r^{2}-2 \theta^{-1}[\bar{a}-\delta-r]}\right)\right] k
$$

Proof: Appendix A.

This maximum amount of borrowing $(-\bar{\eta} k)$ is exogenous to the the firm but endogenous to the model. It increases with the net productivity of firms' assets in the hands of outside investors $(\bar{a}-\delta)$ and decreases with the costs of investing in new capital $(\theta)$ and with the discount rate of outside investors $(r)$ which is also the available rate of interest on cash holding/cash borrowing by the firm. It would be possible to generalise the model by enforcing either a lower exogenous limit on borrowing or a higher exogenous limit on borrowing with an interest rate on borrowing of $\bar{r}(c)>r$ for $c<0$ that compensates lenders for the liquidated value of the firm - that being less than the amount borrowed at liquidation. We do not explore these extensions.

If $c$ reaches this bound then the firm has a choice: either liquidate (in which case its assets are acquired by the lenders and there is no further payment to shareholders); or recapitalise (at a cost to shareholders of $\chi k$ ). 
Proposition 2. Sufficient conditions for an optimal policy for choice of $\left\{i_{t}\right\},\left\{\lambda_{t}\right\},\left\{\epsilon_{\tau}\right\}$ as functions of the single state variable $\eta=c k^{-1}$ to exist and satisfy $i_{t}-\delta<\rho, \forall t$ are

$$
\begin{aligned}
& a-\delta<\rho+\frac{1}{2} \theta \rho^{2}-(\rho-r)\left[1+\theta\left(r-\sqrt{r^{2}-2 \theta^{-1}[\bar{a}-\delta-r]}\right)\right] \\
& \bar{a}-\delta<r+\frac{1}{2} \theta r^{2} .
\end{aligned}
$$

Further, if Equation (4a) is satisfied, the growth rate of the capital stock $g(\eta)$ and the optimal investment rate $i(\eta)$ always satisfies the constraints

$$
\bar{g}=\left(r-\sqrt{r^{2}-2 \theta^{-1}[\bar{a}-\delta-r]}\right) \leq g(\eta)=i(\eta)-\delta<\rho .
$$

Proof: Appendix B.

If a solution exists then it is characterised by the following further proposition:

Proposition 3. An optimal policy choice for $\left\{i_{t}\right\},\left\{\lambda_{t}\right\},\left\{\epsilon_{\tau}\right\}$ as functions of the single state variable $\eta=c k^{-1}$, if it exists and takes the following form: (i) making no dividend payments a long as $\bar{\eta}<\eta<\eta^{*}$ for some value $\eta^{*}$ of $\eta$, while making dividend payments at an unlimited rate if $\eta>\eta^{*}$; (ii) investing at a rate

$$
i=\delta+\theta^{-1}(q-1)
$$

where $W(\eta) k$ is the value of $\Omega$ under optimal policy; and $q(\eta)$ representing the valuation of fixed assets by the firm (the cash price it would be willing to pay for a small increase in $k$ ) is given by:

$$
q=\frac{W}{W^{\prime}}-\eta, \quad q^{\prime}=-\frac{W W^{\prime \prime}}{W^{\prime} W^{\prime}}
$$

with $q^{\prime}>0$ whenever $\eta<\eta^{*}$; and $W(\eta)$ is the unique solution to the second-order differential equation over $\eta \in\left[\bar{\eta}, \eta^{*}\right]:$

$$
\rho \frac{W}{W^{\prime}}=a-\delta+r \eta-\frac{1}{2} \sigma^{2}\left(-\frac{W^{\prime \prime}}{W^{\prime}}\right)+\frac{1}{2} \theta^{-1}\left(\frac{W}{W^{\prime}}-\eta-1\right)^{2}
$$

obtained subject to three boundary conditions: (i) an optimality condition for payment of dividends at $\eta^{*}$ $W^{\prime \prime}\left(\eta^{*}\right)=0$ (ii) a scaling condition $W^{\prime}\left(\eta^{*}\right)=1$; and (iii) the matching condition:

$$
W(\bar{\eta})=\max \left[W\left(\eta^{*}\right)-\left(\eta^{*}-\bar{\eta}+\chi\right), 0\right] .
$$

Finally, the firm recapitalises only on the lower boundary and only if $W(\bar{\eta})>0$ in which case it recapitalises by increasing $\eta$ immediately to $\eta^{*}$.

Proof: Appendix B.

This solution combines barrier control at an upper level of the state variable with either impulse control or absorption at a lower level. 
- Barrier control is applied at an upper level of cash holding/borrowing $\eta^{*} k$, retaining all earnings when below this level and paying out all earnings that would take it beyond this level (a form of barrier control). It never holds more cash (or conducts less borrowing) than this targeted amount, and below this level no dividends are paid (as discussed in Section 2, similar barrier control appears in a number of earlier papers studying corporate decision making subject to external financing constraints).

- Impulse control through recapitalisation at a lower boundary $\bar{\eta} k$, but only if the cost to shareholders of recapitalisation is less than their valuation of the recapitalised firm. Net worth is then restored to the upper impulse control level $\eta^{*} k$. The value of the firm at the lower boundary $W(\bar{\eta})$ is the value at the upper boundary $W\left(\eta^{*}\right)$ less the total costs of recapitalisation $\left(\eta^{*}-\bar{\eta}+\chi\right)$.

- Absorption if instead the cost of recapitalisation at the lower boundary representing the maximum level of borrowing exceeds the valuation of the recapitalised firm. It then liquidates and the value obtained by shareholders is zero.

In the absence of financing constraints (as discussed in Appendix A), impulse control is exerted for all values of $\eta \neq 0$ to immediately enforce $\eta=0$, leverage is no longer relevant to the decisions of the firm (the Modigliani-Miller [16] proposition applies) and the value function is linear in $\eta$ and given by $\Omega=k\left(W_{0}+\eta\right), W^{\prime}=1$ and $q=W / W^{\prime}-\eta=W_{0}$.

The outcome is very different in the presence of financing constraints. The value function is then distorted downward. As $\eta$ declines towards the maximum level of borrowing, an increasing marginal valuation of cash results (the slope of $W$ ) because $W^{\prime \prime}<0$. This increasing marginal valuation of cash as the firm comes closer to liquidation is reflected in a curvature of the value function $\Omega=k W(\eta)$ characteristic of dynamic models of financing constraints (see the upper panel of Figure 1 and discussion in Section 2). See [1] for further discussion).

This higher marginal valuation of cash results in a reduction of $q$, the marginal or the internal cost of cash $\left(\Omega_{c}\right)$ relative to the marginal benefits of capital $\left(\Omega_{k}\right)$. The further $\eta$ falls below the target $\eta^{*}$, the more investment is reduced in order to realise cash and stave off costly liquidation or recapitalisation.

The implications of the model for dynamic behaviour can be analysed using the steady state "ergodic distribution." The ergodic distribution, if it exists, represents both the cross-sectional distribution of many firms subject to independent shocks to cash flow and the unconditional time distribution of a single firm across states. It indicates the relative amount of time in which a firm stays in any particular state. When this is high, it visits this state often; when it is low then it visits this state rarely.

If a firm is liquidated at the lower boundary, i.e., if there is no recapitalisation, and it is not replaced by new firms, then no ergodic density exists. In order to compute an ergodic distribution and for comparability with the case of recapitalisation, an additional assumption is required: that liquidated firms are replaced at the upper dividend paying boundary. The following proposition then applies:

Proposition 4. The pdf of the ergodic distribution is described the following first-order ODE:

$$
\frac{1}{2} \sigma^{2} f^{\prime}-\left[a+r \eta-\delta-\theta^{-1}(1+\eta)(q-1)-\frac{1}{2} \theta^{-1}(q-1)^{2}\right] f=-d .
$$

and can be computed subject to the boundary conditions

$$
f(\bar{\eta})=0
$$

and $F\left(\eta^{*}\right)=1$ where $F(\eta)=\int_{u=\bar{\eta}}^{\eta} f(u) d u$. 
Proof: Appendix B.

Here $d$ is a constant representing the net flow of companies through the non-dividend paying region, until they exit at the lower boundary $\bar{\eta}$ through liquidation or recapitalisation and are replaced at the upper boundary $\eta^{*}$.

The interpretation of this ergodic distribution is slightly different in the two cases of recapitalisation and of liquidation. In the case of recapitalisation this represents the steady state cross-sectional distribution of firm net worth for firms hit by independent shocks and the proportion of time spent by a firm at each level of net worth. In the case of liquidation, it represents only the cross-sectional distribution of firm net worth and only when liquidated firms are indeed replaced at the upper boundary. (Other replacement assumptions are possible, for example, replacement at different levels of net worth in proportion to the steady state distribution of firms in which case the right-hand side of Equation (8) is replaced by $-d f$. The ergodic density still represents only a cross-sectioaln distribution).

\subsubsection{Numerical Solution}

Appendix $C$ presents the methods of numerical solution. The outline of these is as follows, utilising the function $q(\eta)$. Equation (6) can be written as:

$$
q^{\prime}=\frac{2}{\sigma^{2}}\left[a-\delta-(\rho-r) \eta-\rho q+\frac{1}{2} \theta^{-1}(q-1)^{2}\right](q+\eta) .
$$

requiring only two boundary conditions for solution: the optimality condition locating the upper boundary $q^{\prime}\left(\eta^{*}\right)=0$ together with the condition on the lower boundary Equation (7).

In the case of liquidation no iteration is necessary. This is because $W(\bar{\eta})=0$ implying from Equation (5) that $q(\bar{\eta})=-\bar{\eta}$, i.e., the maximum amount of lending is the valuation of capital by outsiders and this determines the value of $q$ on the lower boundary. Equation (10) is simply computed directly beginning from the lower boundary with $q=-\eta$ and continuing for higher values of $\eta$ until $q^{\prime}=0$ and the upper boundary, if it exists, is located.

Iteration is required when there is recapitalisation rather than liquidation. This is because in this case $q(\bar{\eta})$ is not known, but must be determined from the matching condition $W(\bar{\eta})=W\left(\eta^{*}\right)-$ $\left(\eta^{*}-\bar{\eta}+\chi\right)$. Given any initial starting value for $q(\bar{\eta})$ it is possible to jointly compute both $q(\eta)$ and the accompanying value function $W(\eta)$. Iteration on the starting value $q(\bar{\eta})$ then yields the solution with recapitalisation (if one exists) with $W(\bar{\eta})>0$. While numerical solution is straightforward, it may fail to locate an upper boundary $\eta^{*}$ for some combinations of parameters. This happens, for example, when the productivity of capital $a$ is so high, and the adjustment costs of capital increase $\theta$ are so low, that output can be reinvested to increase the stock of capital faster than the discount rate of firms. (See Appendix A) for a discussion of the parameter restrictions required to prevent this in the deterministic case $\sigma=0)$. In this case the value function is unbounded and there is no meaningful solution. Extreme parameter values, for example, very low values of $\sigma$, can also result in numerical instability and failure to find a solution.

\subsection{Simulation Results}

Numerical solution is rapid, allowing extensive simulations of the model equations. Focusing on the shape of the ergodic distribution $f(\eta)$, one question is whether it has two peaks and can therefore help explain a transition from a high output boom to a low output slump, or instead has a single peak. In this first version of the model in this section there is always a single peak located at the maximum value $\eta^{*}$, i.e., the model without rental or sale of capital does not create long lasting periods with output and investment below normal levels. 
Typical value functions $W$ together with the corresponding ergodic densities $f$ are presented in Figure 1. For that, the chosen parameters were:

$$
\begin{gathered}
\rho=0.06, \quad r=0.05, \quad \sigma=0.2 \\
\theta=15.0, \quad \chi=0.75 \\
a=0.1, \quad \bar{a}=0.04, \quad \delta=0.02 .
\end{gathered}
$$

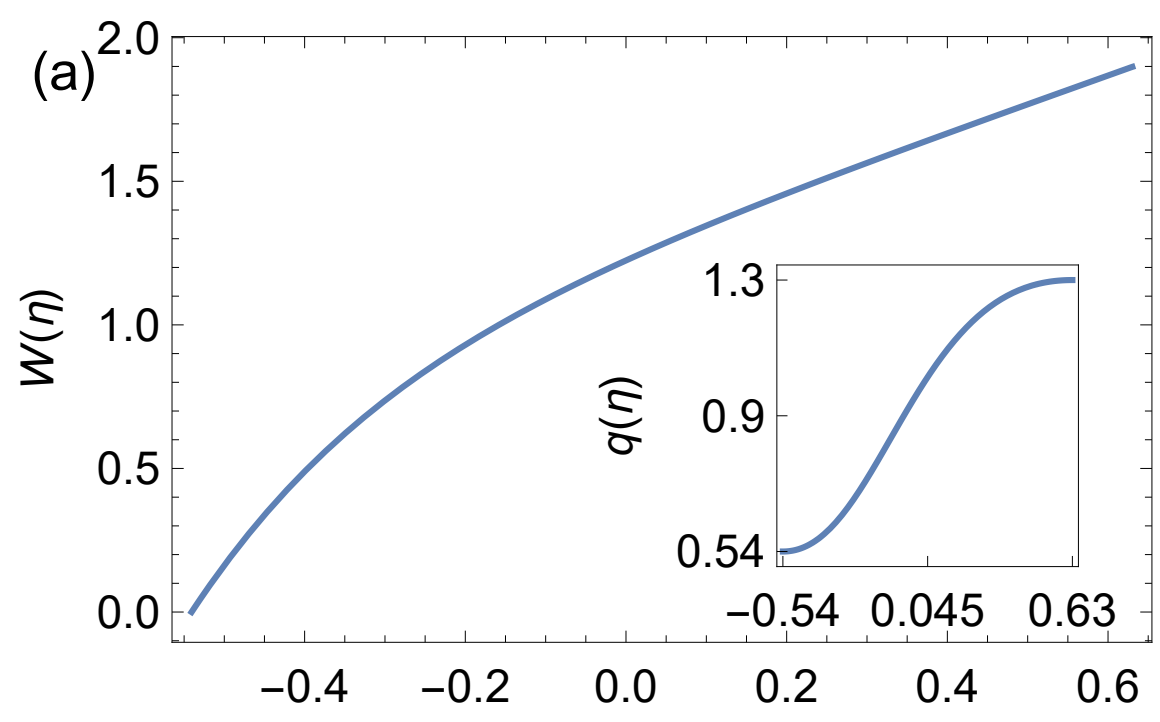

$n$

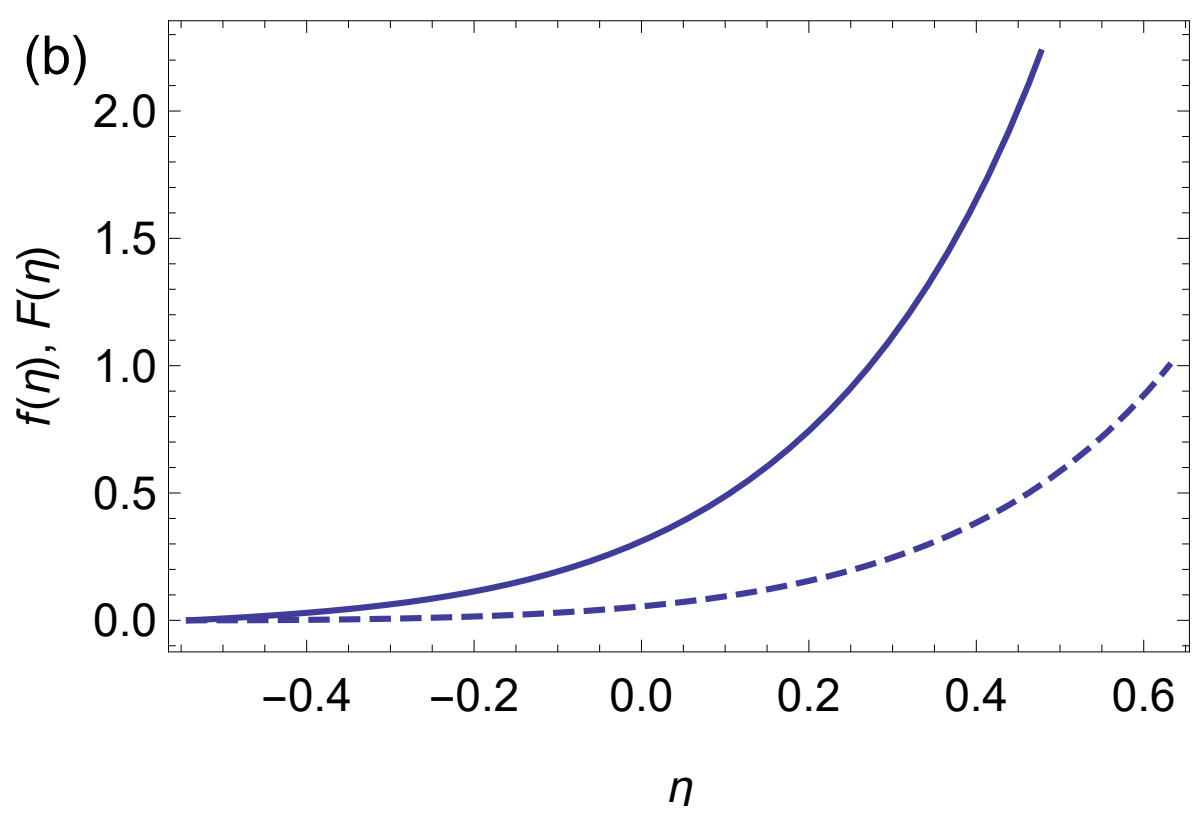

Figure 1. Solutions of the model equations of Section 3 for baseline parameters $\rho=0.06, r=0.05$, $\sigma=0.2, a=0.1, \bar{a}=0.04, \delta=0.02, \theta=15$ and $\chi=0.75$. Subfigure (a): value function $W$, inset shows the function $q$ over the same $\eta$ range; (b) the ergodic density $f$ (solid curve) and the cumulative density function $F$ (dashed).

Note the shape of these plots, with a monotonically increasing value function $W$, and a single-peaked ergodic density with a maximum at $\eta^{*}$. Across a wide range of parameter space 
search, only single-peaked distributions of this kind emerge. Although double peaks were not found, in some simulations the main peak normally at $\eta^{*}$ can migrate into the central part of the $\eta$ range. This occurs when choosing parameters for which cash-flows are non-positive $(\mathrm{d} \eta \leq 0)$. We do not report these simulations here.

\section{An Extended Model}

This section extends the model of Section 4 by assuming that capital can be rented by firms to outside investors. The structure of this section parallels that of Section 3, with subsections on assumptions (Section 4.1), the solution (Section 4.2) and simulation results (Section 4.3).

\subsection{Additional Assumptions}

In this extended setting, firms continue to manage the same two "state" variables, net cash $c$ and capital $k$, but these now evolve according to:

$$
\begin{aligned}
\mathrm{d} c= & \left.-\lambda+[\psi a+(1-\psi) \bar{a}] k+r c-i k-\frac{1}{2} \theta(i-\delta)^{2} k\right\} \mathrm{d} t \\
& +\epsilon(\tau)+\psi \sigma_{1} k \mathrm{~d} z_{1} \\
\mathrm{~d} k= & (i-\delta) k \mathrm{~d} t+\psi \sigma_{2} k \mathrm{~d} z_{2} .
\end{aligned}
$$

There are now two independent diffusion terms $\left(\psi \sigma_{1} k \mathrm{~d} z_{1}\right.$ and $\left.\sigma_{2} \psi k \mathrm{~d} z_{2}\right)$ and an additional third control variable, the proportion of capital $\psi$ firms themselves manage (with remaining capital $1-\psi$ rented to households). All the other assumptions of Section 3 continue to apply.

Due to competition amongst households to acquire this capital, the amount households are willing to pay and hence the income from renting out a unit of capital is $\bar{a}$ the productivity of capital when managed by households. A special case is when $\bar{a}=0$. In this case the renting of capital can be understood as "mothballing," taking a proportion $1-\psi$ capital out of production. In either case, whether renting or mothballing, the firm benefits from a reduction in the diffusion terms from $\sigma k$ to $\psi \sigma k$, protecting it from the risk of fluctuations in net worth.

The introduction of a second diffusion term is a modest extension of the model. This introduces a dependency of diffusion on the level of net worth with $\sigma^{2}(\eta)=\sigma_{1}^{2}+\sigma_{2}^{2} \eta^{2}$ instead of a constant $\sigma^{2}$. The introduction of renting is a more fundamental change, leading to the possibility of a double-peaked ergodic density and the possibility of persistence of a sequence of negative shocks that push net worth down to very low levels (the "net worth trap").

\subsection{Solution}

\subsubsection{Characterisation of Solution}

Propositions 1 and 2 apply to the generalised model with renting. Proposition 3 applies in the following amended form:

Proposition 5. An optimal policy choice for $\left\{i_{t}\right\},\left\{\psi_{t}\right\},\left\{\lambda_{t}\right\},\left\{\epsilon_{\tau}\right\}$ as functions of the single state variable $\eta=c k^{-1}$, if it exists, takes the following form. The rules for $i(\eta), \lambda(\eta)$ are exactly as stated in Proposition 3; optimal policy for $\psi(\eta)$ renting of fixed capital is that for lower values of $\eta$, in the range $\bar{\eta} \leq \eta<\tilde{\eta}$ where $\bar{\eta} \leq \tilde{\eta}<\eta^{*}$, firms retain a proportion $\psi<1$ of fixed capital given by:

$$
\psi=\frac{a-\bar{a}}{\sigma^{2}(\eta)}\left[-\frac{W^{\prime \prime}}{W^{\prime}}\right]^{-1}
$$


and rent the remaining proportion $1-\psi>0$ to firms; and for $\eta \geq c$, firms retain all fixed capital, i.e., $\psi=1$ and none is rented out. Here $\sigma^{2}(\eta)=\sigma_{1}^{2}+\sigma_{2}^{2} \eta^{2} W(\eta)$; the unique solution to the second-order differential equation over $\eta \in\left[\bar{\eta}, \eta^{*}\right]$, now obeys:

$$
\begin{gathered}
\rho \frac{W}{W^{\prime}}=\bar{a}+(a-\bar{a}) \psi-\delta+r \eta-\frac{\sigma^{2}(\eta)}{2} \psi^{2}\left[-\frac{W^{\prime \prime}}{W^{\prime}}\right] \\
+\frac{1}{2 \theta}\left[\frac{W}{W^{\prime}}-1-\eta\right]^{2}
\end{gathered}
$$

Solution for $W$ is found subject to same boundary conditions as in Proposition 3

Proof: Appendix B

Here $-W^{\prime \prime} / W^{\prime}$ expresses the induced risk aversion created by the presence of financing constraints ( Section 4 of [1] has further discussion of this induced risk aversion and a comparison with the risk loving behaviour that emerges in many standard discrete time models as a result of moral hazard). $-W^{\prime \prime} / W^{\prime}$ appears also in Proposition 3, the solution for the model with no option to rent out capital. There though, while it appears in Equation (6) the second-order differential equation for the value function, it has no direct impact on firm decisions. Now in Proposition 5, induced risk aversion $-W^{\prime \prime} / W^{\prime}$ has a direct impact on firm decisions once net worth $\eta$ falls below $\tilde{\eta}$. Renting out productive capital to households then reduces both the drift and the diffusion of $\eta$.

The introduction of the option to rent out capital introduces a second component to the behaviour of the firm. Now, and with the reduction of investment in the basic model because of a higher internal cost of capital, they can also reduce their employment of capital as a response to higher induced risk aversion. As a consequence of reduction in the employment of capital, in effect a "shrinking" of the size of operations, the firm can get "stuck" near the bankruptcy threshold, leading to a second peak in the ergodic distribution.

The resulting ergodic density can be computed using this Proposition (an indirect statement is used because of the dependency of $\psi$ and $\sigma$ on $\eta$. While $\phi$ can be substituted out from Equation (15) the resulting ODE for $f$ is rather cumbersome):

Proposition 6. The pdf of the ergodic distribution is described by the following first-order ode:

$$
\phi^{\prime}-\left[\frac{1}{2} \psi^{2} \sigma^{2}(\eta)\right]^{-1}\left[a+(a-\bar{a}) \psi+r \eta-\delta-\theta^{-1}(1+\eta)(q-1)-\frac{1}{2} \theta^{-1}(q-1)^{2}\right] \phi=-d
$$

where $\phi=\psi^{2} \sigma^{2} f / 2$ and satisfies the boundary conditions

$$
\left\{\begin{array}{cc}
f(\bar{\eta})=0, & \text { if } W(\bar{\eta})>0 \\
d=0 & \text { if } W(\bar{\eta})=0
\end{array}\right.
$$

and $F\left(\eta^{*}\right)=1$ where $F(\eta)=\int_{u=\bar{\eta}}^{\eta} f(u) d u$.

Proof: Appendix B. 


\subsubsection{Numerical Calculation}

Numerical solution methods are again detailed in Appendix C. This proceeds in the same way as for the first model without renting of Section 3, by re-expressing Equation (14) as a differential equation in $q$. Over the lower region $\eta<\tilde{\eta}$ ( Equation (14)) becomes:

$$
q^{\prime}=-\frac{1}{2} \frac{(a-\bar{a})^{2}}{\sigma_{1}^{2}+\eta^{2} \sigma_{2}^{2}} \frac{q+\eta}{\bar{a}-\delta+r \eta-\rho(q+\eta)+\frac{1}{2} \theta^{-1}(q-1)^{2}}
$$

while in the upper region Equation (10) continues to apply (except that now $\sigma^{2}=\sigma_{1}^{2}+\sigma_{2}^{2} \eta^{2}$ is a function of $\eta$ ).

If there is no recapitalisation then the model can again be solved without iteration, commencing the calculation at $\eta=\bar{\eta}$ and continuing until the intermediate values $\eta=\tilde{\eta}$ and $\eta=\eta^{*}$ are located. However, in this case $q(\bar{\eta})=-\bar{\eta}$ and hence $\psi(\bar{\eta})=0$, with the consequence that there are singularities in $f, q$ and $W$ at $\bar{\eta}$. We incorporate these singularities using asymptotic approximations summarised in the following further proposition.

Proposition 7. $W, q$ and $\phi$ close to $\bar{\eta}$ are described by:

$$
W=C_{W}(\eta-\bar{\eta})^{\beta}(1+\mathcal{O}(\eta-\bar{\eta}))
$$

where $C_{W}$ is a constant and $\beta=1 /\left(1+q^{\prime}(\bar{\eta})\right) \in(0,1]$;

$$
q=\bar{q}+q^{\prime}(\bar{\eta})(\eta-\bar{\eta})
$$

and;

$$
\phi=C_{\phi}(\eta-\bar{\eta})^{\alpha}
$$

where $\alpha$ is given by Equation (A34) of Appendix $D$ and $C_{\phi}$ is another constant.

This further implies that $-W^{\prime \prime} / W^{\prime}$ (our measure of induced risk aversion) is divergent at $\bar{\eta}=-\bar{q}$,

$$
-\frac{W^{\prime \prime}}{W^{\prime}} \simeq \frac{1-\beta}{\eta-\bar{\eta}}
$$

(consistent with $\psi(\bar{\eta})=0$ ), and the ergodic density is approximated by

$$
f \propto(\eta-\bar{\eta})^{\alpha-2}
$$

and thus diverges if $\alpha<2$ and becomes degenerate, with the entire probability mass at $\bar{\eta}$ if $\alpha \leq 1$.

Proof: Appendix D.

In the case of recapitalisation $q(\bar{\eta})>-\bar{\eta}$ and there is are no singularities in the solution; so, while iteration ia again required to determine $q(\bar{\eta})$, this can be conducted in exactly the same way as described in Section 3.2.2 for the model without renting. 


\subsection{Simulation Results}

As expected from the power-law shape of $f$, Equation (21), the option to rent can have a strong impact on the shape of the ergodic density. As an example of this, in Figure 2 plots the value function $W$ together with $q$, and the probability and cumulative densities, now using baseline parameters $\rho=0.06$, $r=0.05, \sigma_{1}=0.2, \sigma_{2}=0.0, a=0.1, \bar{a}=0.04, \delta=0.02, \theta=15$ and $\chi=0.75$ (identical parameters to those used in Figure 1). Whereas the value function $W$ and $q$ show little change when renting is introduced, the density function $f$ changes dramatically. This time a second peak is clearly present near the left-hand side range of $\eta$ values. Note that with these particular parameter values the firm chooses to recapitalise, with $\chi$ being slightly less than a critical value of around 0.55 at which recapitalisation is not worthwhile, and $W(\bar{\eta}) \approx 0.05$. The interested reader can observe, using our standalone application, how increasing $\chi$ to above this critical level results in the emergence of singularities and the divergence of $f(\eta)$ to $+\infty$ at $\eta=\bar{\eta}$.
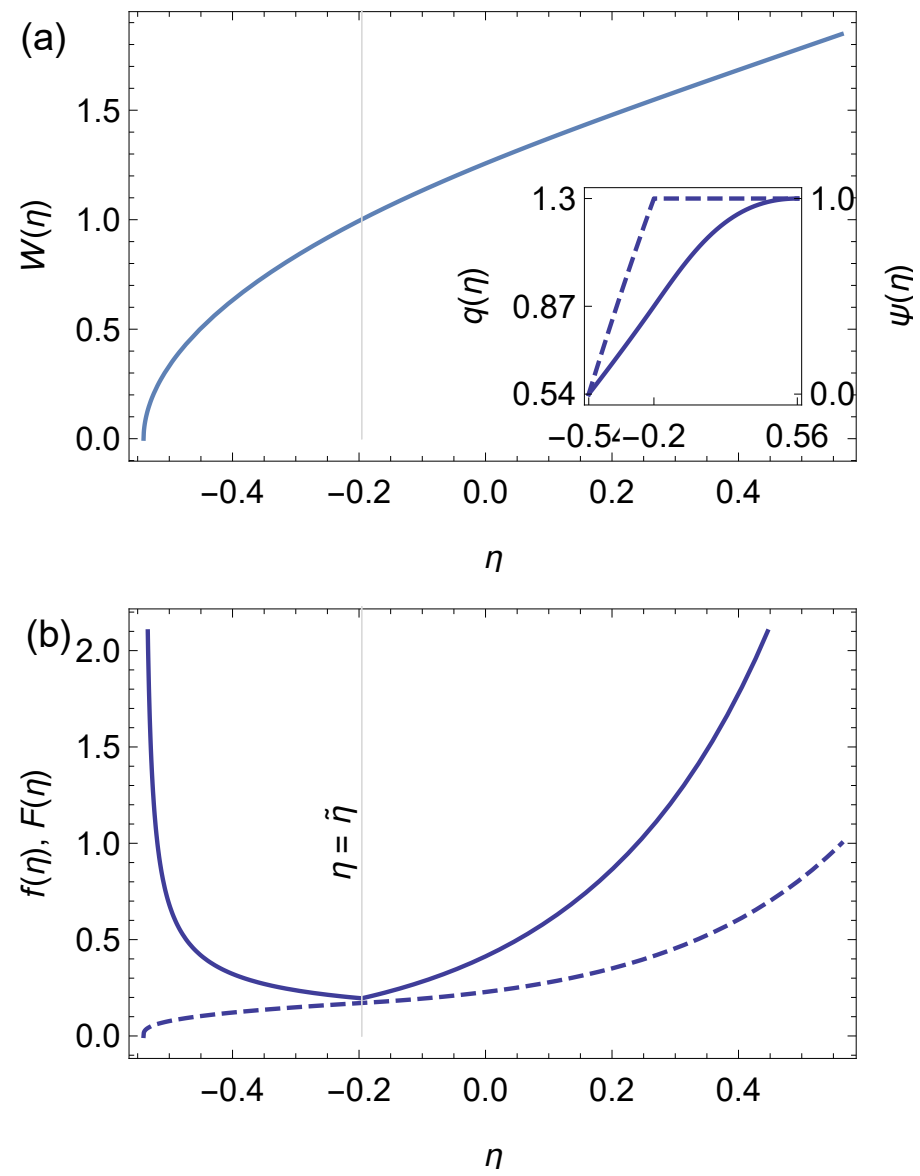

Figure 2. Solutions of the model of Section 4 with option to rent, using baseline parameters $\rho=0.06$, $r=0.05, \sigma_{1}=\sigma=0.2, \sigma_{2}=0.0, a=0.1, \bar{a}=0.04, \delta=0.02, \theta=15$ and $\chi=0.75$. Contrast this to Figure 1 where identical parameters were used, but without renting. Subfigure (a): value function $W$, inset shows the functions $q$ and $\psi$ over the same $\eta$ range; (b) the ergodic density $f$ (solid curve) and the cumulative density function $F$ (dashed). Notice the prominent peak in $f$ towards the left-hand side boundary.

This ergodic instability (a second peak towards in the ergodic density associated with low values of the state variable $\eta$ representing the ratio of cash-to-capital) is parameter dependent. This parameter dependence emerges in two different ways: (i) through the power-law exponent $\alpha$, and (ii) dependence on the cost of recapitalisation $\chi$. The ability to recapitalise or not has a major impact on the ergodic distribution. For any given parameters, there is a threshold $\chi, \bar{\chi}$, above which recapitalisation is no longer worthwhile. If $\chi$ is equal to or greater than this value, then $\psi(\bar{\eta})=0$, and the density diverges 
and the ergodic density follows the power-law $f \propto(\eta-\bar{\eta})^{\alpha-2}$ near $\bar{\eta}$, which in turn can lead to infinite densities. Hence, the strength of the instability (i.e. the amount of probability mass near $\bar{\eta}$ ) is strongly controlled by the parameter $\chi$.

This is illustrated in Figure 3 showing how the ergodic density changes as $\chi$ is varied. For low values of $\chi$, there is no left-hand side peak in the model with rent (Figure 3a) and $f$ largely resembles that of the model without the option to rent (Figure 3b). As $\chi$ approaches $\bar{\chi}$ (indicated by the dotted lines on the floor of the two panels of this figure, where $\bar{\chi} \simeq 0.55$ with rent, $\bar{\chi} \simeq 0.54$ without), in the model with renting a probability mass starts to appear near $\bar{\eta}$. Crossing $\bar{\chi}$, recapitalisation becomes no longer an option, and the density at $\bar{\eta}$ diverges. Above $\bar{\chi}$ there is no longer $\chi$ dependence. Note that the distribution $f$ changes quite sharply; when approaching $\bar{\chi}$ is crossed, with a second peak of the distribution emerging close to $\eta=\bar{\eta}$, a robust result across a variety of simulations.

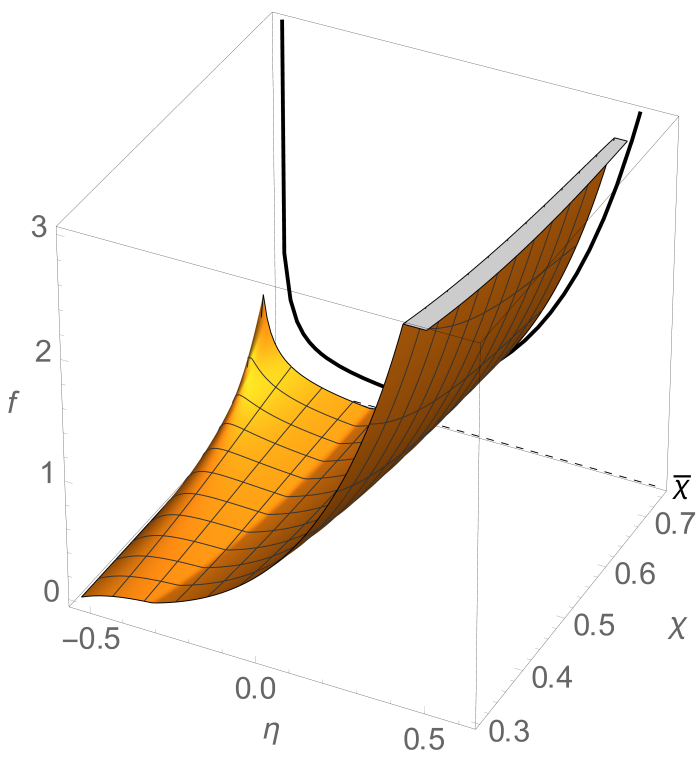

(a)

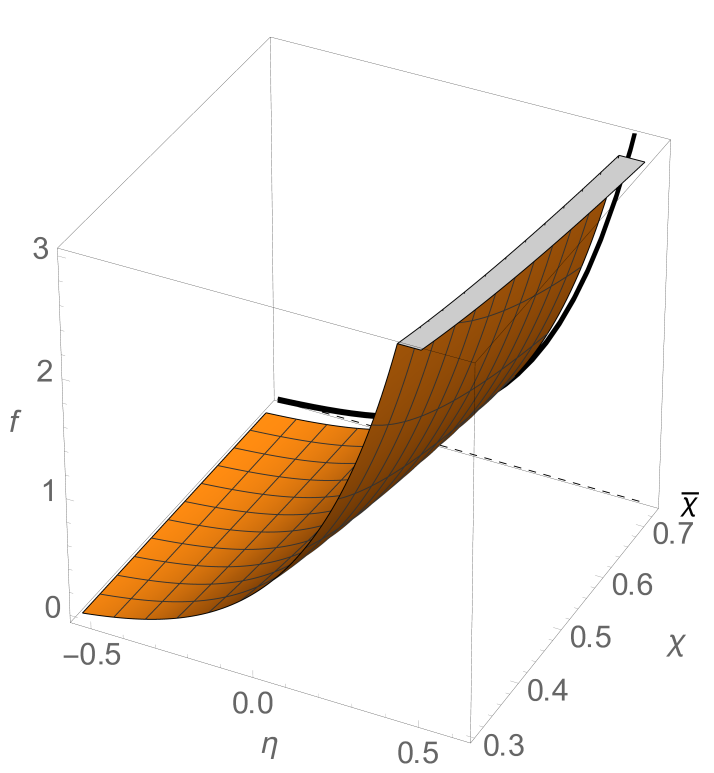

(b)

Figure 3. Comparison of ergodic densities $f$ given the option to rent (a) and no option to rent (b) as the financing constraint $\chi$ is varied. Other parameters were set to baseline values. The lower boundary is recapitalising unto $\chi=\bar{\chi}(\bar{\chi} \simeq 0.64$ in (a) and 0.62 in (b)), indicated by the thick solid line on the graph and dashed line on the axis. In (a) a left-boundary peak emerges for $\chi$ just less than $\bar{\chi}$. Density is infinite at $\bar{\eta}$ for $\chi>\bar{\chi}$. Note the complete absence of the left-hand side peak in (b).

To further explore this parameter dependence consider how the median of $f$ depends on various parameters. Since the values of $\bar{\eta}$ and $\eta^{*}$, the range on which the distribution is defined, also vary with the parameters, it is convenient to scale the median on to the interval $[0,1]$ : Let $m$ be the median; then the scaled median is defined as

$$
\tilde{m}=\frac{m-\bar{\eta}}{\eta^{*}-\bar{\eta}^{\prime}}, \quad F(m)=\frac{1}{2}
$$

A value of $\tilde{m} \sim 0$ implies that most of the probability mass is concentrated near $\bar{\eta}$, while $\tilde{m} \sim 1$ suggests that firms are more probably found near $\eta^{*}$. While this is a somewhat crude measure (e.g., the median cannot distinguish between distributions that are $\cup$ or $\cap$-shaped), nonetheless, $\tilde{m} \lesssim 1 / 2$ is a strong indicator of large mass of probability near the lower boundary, hence the long lasting response to a large initial shock found by [2].

In Figure 4 presents a contour plot $\tilde{m}$ as a function of the financing constraint $\chi$ and the volatility $\sigma$ (note that Figure 3 represents a small slice of data presented in this figure). The solid heavy line represents the critical value $\bar{\chi}(\sigma)$, the firm choosing to recapitalise only when $\chi<\bar{\chi}(\sigma)$. Three roughly distinct regimes can be seen: 
(i) The low volatility range $\sigma \lesssim 0.2$, in which the firm always prefers to recapitalise and where $\tilde{m} \gtrsim 0.8$ and so most of the probability is found near the dividend paying boundary.

(ii) A region where $\sigma \gtrsim 0.3$ and at the same time $\chi \gtrsim 0.5$, i.e., red region to the top right, where $\tilde{m} \sim 0$, and much of the probability mass is located near the left-hand boundary.

(iii) An intermediate transition range wherein small changes in either $\sigma$ or $\chi$ result in a very substantial change in $\tilde{m}$. This transition is especially abrupt for high values of $\sigma$.

Exploring the behaviour of $\tilde{m}$ as a function of other model parameters yields remarkably similar contour plots. For example, as the relative impatience of shareholders $\rho-r$ is increased from relatively low to high values, there are also two distinct regions similar to those of Figure 4, with a relatively sharp transition in the balance of the probability distribution from near the upper boundary $\eta^{*}$ to the lower boundary $\bar{\eta}$.

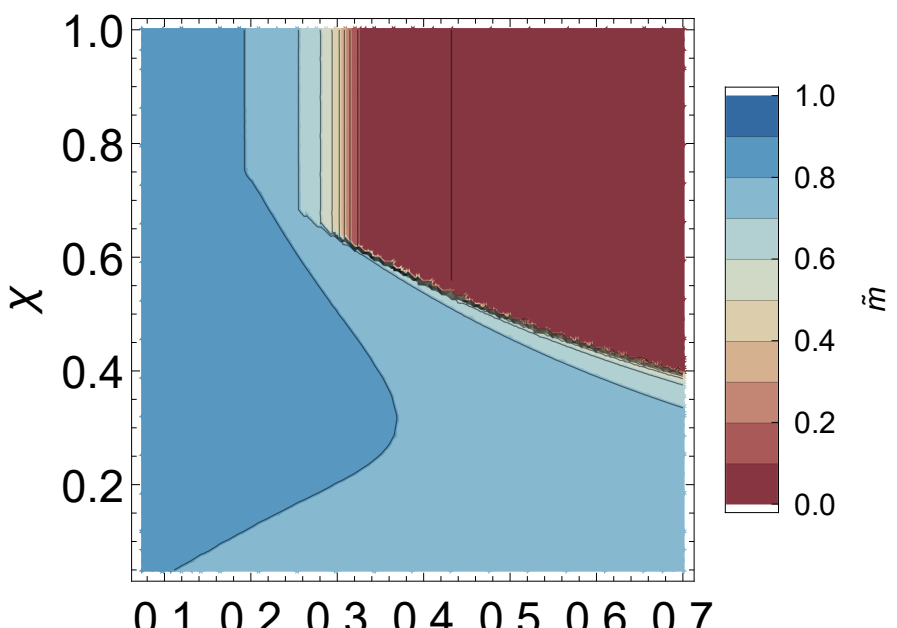

$\sigma$

Figure 4. The scaled median $\tilde{m}$ as a function of $\chi$ and $\sigma . \tilde{m}$ is the median of the distribution of $\eta$ relative to its range. $\tilde{m}$ close to one indicates that the mass of the distribution is located near the upper dividend paying boundary. $\tilde{m}$ close to zero indicates that the mass is located near the lower liquidation or recapitalisation boundary. $\sigma_{2}=0.0$ (the baseline value from earlier figures) so $\sigma_{1}=\sigma$. Other parameters were set to baseline values; $\rho=0.06, r=0.05, a=0.1, \bar{a}=0.04, \delta=0.02$ and $\theta=15$. Contours are plotted at level values of $\tilde{m}$ and are spaced at intervals of 0.1 .

One further finding concerns induced aversion to cash flow risk $-\frac{W^{\prime \prime}}{W^{\prime}}$. This induced risk aversion is, like ergodic instability, strongly parameter and model structure dependent. In the model with renting, when firms do not recapitalise they become extremely risk-averse close to the lower boundary $\bar{\eta}$. This is revealed by an analysis of power-law behaviour of $W$ at the lower boundary $\bar{\eta}$ (see Proposition 7). This extreme risk aversion does not arise in the model with renting or if recapitalisation is not costly.

This finding is illustrated in Figure 5 which compares induced risk-aversion for the two versions of the model, with and without the option to rent. The parameters here are the same as in Figures 1 and 2. For relatively large values of $\eta$ close to $\eta^{*}$ the option to rent provides protection against cash flow risk and induced risk aversion $-\frac{W^{\prime \prime}}{W^{\prime}}$ is lower for the model with renting; but as $\eta$ falls down towards $\bar{\eta}$, the model with renting induced risk aversion $-\frac{W^{\prime \prime}}{W^{\prime}}$ diverges upwards-rising increasingly rapidly as $\eta$ approaches $\bar{\eta}$, whereas it rises only slightly in the model without renting. 


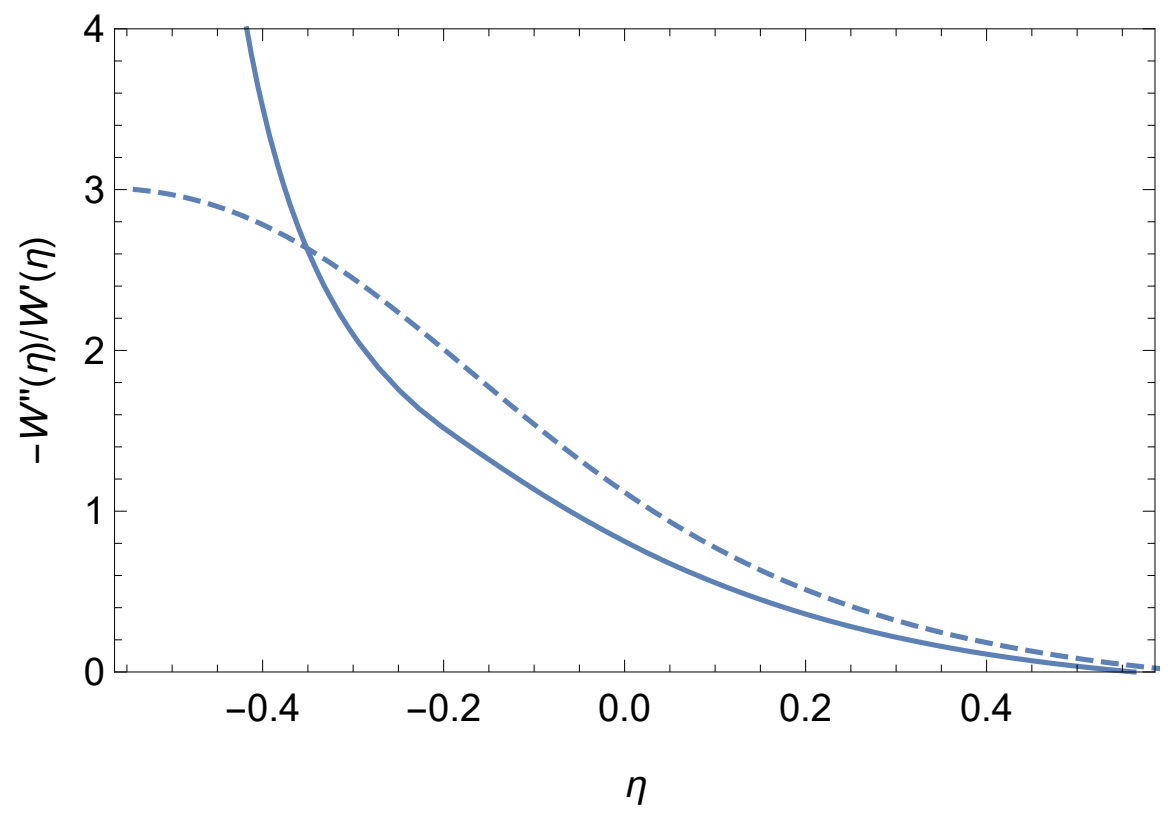

Figure 5. Induced risk aversion $-W^{\prime \prime} / W^{\prime}$ as a function of $\eta$. Parameters were set to baseline. Solid curve: model with option to rent; dashed curve: model without option to rent. Significantly, the risk aversion diverges strongly near $\bar{\eta}$ in the model without renting, in contrast to the model with renting.

\section{Conclusions}

This paper investigated the impact of financing constraints on corporate output and investment in a simple continuous time setting with linear production and preferences. Firms face liquidation or costly recapitalisation, if their net worth (relative to capital) falls to a minimum level following a shock to cash flows or productivity. The boundary conditions resulting from these financing constraints generate potentially large and long-lasting non-linearities in the response of firm output and investment to external shocks, even in this otherwise linear setting. A fall of net worth leads to a decline of investment below normal levels. Moreover, if firms can rent out or mothball their capital stock, output also declines along with investment, and this may continue for an extended period of time: the "net worth trap."

Several further insights emerged. One is the importance of "corporate prudential saving," analogous to the household prudential saving extensively discussed in the literature on the consumption function. As their net worth declines, firms invest less and less (a fall in marginal q). The second is "induced risk aversion": firms with sufficiently high net worth have the same attitude to risk as their share holders (assumed for simplicity to be risk-neutral); but as net worth declines then firms behave increasingly as if they were averse to risk in order to reduce the probability of future liquidation or costly recapitalisation, here by mothballing or renting out more and more of their capital. Figures 4 and 5 show how resulting behaviour can vary markedly (both qualitatively and quantitatively) with parametrisation and model specification.

The finding that financing constraints mean that firm decisions can be highly non-linear functions of their indebtedness means that both the size and direction of shocks matter. This in turn helps to clarify when a linearisation —of the kind routinely employed in new Keynesian DSGE macroeconomic models-provides a reasonable approximation to the fully dynamic optimal behaviour

In normal times when shocks are comparatively small, aggregate behaviour can be sufficiently well captured in standard linearised specifications. This is illustrated by Figures 1a and 2a. In normal times most firms are located near the right-hand sides of these figures, close to the upper dividend payment boundary $\eta^{*}$ where the value function $W(\eta)$ is approximately linear. The reserve of net worth provides and adequate hedge against both aggregate and idiosyncratic risk. Moreover, in normal times, external equity capital can, if necessary, be raised at relatively low cost; i.e., the cost of 
recapitalisation parameter $\chi$ is low. As a result, impulse responses, expressed as a percentage of initial shock are then approximately the same regardless of the size or direction of the initial disturbance; linearisation of impulse responses based on past data provides a convenient and reliable summary of macroeconomic behaviour.

In times of extreme financial and economic stress, such as the Great Depression of the 1930s or the Global Financial Crisis of 2007-2008, the situation can be quite different. Uncertainty $\sigma$ and the cost of recapitalisation $\chi$ rises. Large shocks push many firms towards the lower minimum level of net worth $\bar{\eta}$, where as illustrated in Figures $1 \mathrm{a}$ and $2 \mathrm{a}$, the value function $W(\eta)$ is concave not linear and both corporate prudential saving and induced risk aversion emerge. Impulse responses based on past data are no longer a reliable guide to the response to shocks. As our Figure 4 illustrates, such an adverse change in the economic environment can lead to a "phase change," with a shift to a regime where the net worth trap emerges. The response to the Covid-19 pandemic may provide another such episode.

Most striking here, as illustrated in our Figure 4, is that relatively small parameter changes can lead to this phase change. A small increase in perceived uncertainty (our parameter $\sigma$ ) leads to a large change in behaviour, from relatively rapid rebuilding of physical and financial capacity following the emergence of financial distress to a slow rebuilding with a long lasting period of reduced output and investment. Small changes in the external environment faced by firms can lead to the emergence of the persistent "net worth trap." Policy makers and regulators need to be aware that whilst linear approximations may provide a good description of usual events, they can give misleading insights in more turbulent times.

Author Contributions: The mathematical modelling, proofs and the selection of illustrative figures reported in the paper are the joint work of all the authors. J.I. conducted the numerical solution and wrote most of the supporting Mathematica code, including the free standing numerical solver. A.M. and D.R. wrote the review of the literature and the economic interpretation of the model's results. All authors have read and agreed to the published version of the manuscript.

Funding: This research received no external funding.

Acknowledgments: The authors are grateful for comments from Jussi Keppo, Feo Kusmartsev, Tassos Malliaris, Jean-Charles Rochet and Javier Suarez; and for feedback from audiences at the Bank of England, the Bank of Finland, the IBEFA January 2013 meetings, IFABS 2014 meeting, the University of Durham, Bristol University, the London School of Economics, the Bank of Japan, the National University Singapore, the University of Tasmania and internal seminar and conference presentations at Loughborough University. Remaining shortcomings are our responsibility alone.

Conflicts of Interest: The authors declare no conflict of interest.

\section{Appendix A. Solution in the Absence of the Non-Negativity Constraint on Dividends}

This appendix considers the solution to the model of this paper in the baseline case wherein dividend payments can be negative, or equivalently, there is no uncertainty. This provides a benchmark for studying and solving the case of the constrained firm for which there is uncertainty and dividends are required to be non-negative. It also yields a convenient formula for the maximum amount of borrowing provided by households to firms.

A crucial intuition emerges from this benchmark model, relevant to the model with a non-negativity constraint on dividend payments. The rate of growth preferred by firms is an increasing function of the ratio of debt to capital (this is because debt increases at the same rate of growth of capital, creating an additional cash flow that can be used for investment, and the higher the ratio of debt to capital, the greater this cash flow). If the financing constraint is sufficiently lax then it is possible for firms to achieve a growth rate equal to their own rate of discount while still being able to pay dividends. In this case the objective of this firm (expected discounted dividend payments) 
is unbounded and the solution is no longer meaningful. Therefore, some financing constraint is required in order for the model to have a meaningful solution.

To solve this benchmark, note that since firm owners can freely transfer funds into or out of the firm, optimal policy is to maintain the ratio of cash balances $\eta=c / k$ at whatever rate is preferred by borrowers, subject to the highest level of indebtedness allowed by lenders $\eta \geq \bar{\eta}$. If the initial time $t=0$ ratio $\eta_{0}$ differs from the desired ratio $\eta$ then an instantaneous dividend payment of $\left(\eta_{0}-\eta\right) k$ is immediately made to bring the cash to capital ratio to the desired value of $\eta$.

There is therefore now only a single state variable $k$. The value function (the value of the objective function under optimal policy) is linearly homogeneous in $k$ and so can be written as $V=k W$, where $W$ is a constant that depends on the parameters representing preferences and the evolution of the state variable $k$. This in turn implies that $V_{k}=W$ and $V_{k k}=0$. Expected dividend payments will be determined by the expected net cash flow of the firm plus any additional borrowing possible because $k$ and hence $c$ are growing. The remaining policy decision is to choose a rate of investment $i$ and hence expected growth of the capital stock $g=i-\delta$ to maximise $\Omega$, Equation (2)

The solution can be summarised in the following proposition.

Proposition A1. Assuming $\rho>r$, then an optimal policy yielding positive pay-offs for the owners of the firm can be found provided that:

$$
-2 \frac{\rho-(a-\delta)+(\rho-r) \bar{\eta}}{\rho^{2}}<\theta< \begin{cases}\infty & \text { if } a+r \bar{\eta} \geq \delta \\ \frac{1}{2} \frac{(1+\bar{\eta})^{2}}{\delta-r \bar{\eta}-a} & \text { if } a+r \bar{\eta}<\delta\end{cases}
$$

in which case an instantaneous dividend payment of $\eta_{0}-\bar{\eta}$ is made so that $\eta=\bar{\eta}$, the growth rate of the capital stock is constant (state independent) and is given by:

$$
g=\rho-\sqrt{\rho^{2}-2 \theta^{-1}[a-\delta-\rho+(r-\rho) \bar{\eta}]}<\rho,
$$

while the value of the maximised objective is given by:

$$
V\left(\eta_{0}, k\right)=\left(\eta_{0}-\bar{\eta}\right) k+\frac{(a-\delta)+(r-g) \bar{\eta}-g-\frac{1}{2} \theta g^{2}}{\rho-g} k=\left(1+\eta_{0}+g \theta\right) k
$$

where $\left[(a-\delta)+(r-g) \bar{\eta}-g-\frac{1}{2} \theta g^{2}\right] k$ is the expected flow of dividends per period of time paid to shareholders.

Proof. The firm has two choice variables, $\eta$ and $g$ (with investment expenditure given by $i k=(g+\delta) k$ and associated quadratic adjustment costs of $\left.\frac{1}{2} \theta(i-\delta)^{2}=\frac{1}{2} \theta g^{2}\right)$. The equations of motion (1) still apply and dividends are paid according to:

$$
\lambda \mathrm{d} t=\left[(a-\delta)+(r-g) \eta-g-\frac{1}{2} \theta g^{2}\right] k \mathrm{~d} t+\sigma k \mathrm{~d} z
$$

Substituting for $\lambda$ the discounted objective can be written as:

$$
\Omega=\max _{\eta, g}\left\{\mathbb{E} \int_{0}^{\infty} \mathrm{e}^{-\rho t}\left[(a-\delta)+(r-g) \eta-g-\frac{1}{2} \theta g^{2}\right] k \mathrm{~d} t+\left(\eta_{0}-\eta\right) k_{0}+\int \mathrm{e}^{-\rho t} \sigma k \mathrm{~d} z\right\}
$$

yielding, since $\mathbb{E}[k]=k(0) \exp (g t)$ and $\mathrm{e}^{-\rho t} \sigma k=0$ :

$$
\Omega=k(0) \max _{\eta, g}\left[\eta_{0}-\eta+\frac{(a-\delta)+(r-g) \eta-g-\frac{1}{2} \theta g^{2}}{\rho-g}\right] .
$$


The growth rate $g$ that maximises the right-hand side of this expression is determined by the first-order condition with respect to $g$ :

$$
\frac{1}{2} g^{2}-\rho g-\theta^{-1}[\rho-(a-\delta)+(\rho-r) \eta]=0
$$

yielding the solution (the positive root of the quadratic can be ruled out because $g<\rho$ to ensure that the value function is finite and that the second-order condition for maximisation is satisfied):

$$
g=\rho-\sqrt{\rho^{2}+2 \theta^{-1}[\rho-(a-\delta)+(\rho-r) \eta]} .
$$

Writing $\rho-g=\sqrt{\rho^{2}+2 \theta^{-1}[\rho-(a-\delta)+(\rho-r) \eta]}=R$, implying $g^{2}=\rho^{2}-2 \rho R+R^{2}$, and substitution into Equation (A5) then yields: (A3).

The indebtedness is determined by the first-order condition in (A5) with respect to $\eta$ :

$$
\frac{r-g}{\rho-g}-1=\frac{r-\rho}{\rho-g}<0
$$

establishing that the firm will seek to borrow as much as it possibly can. Hence, the firm will make an instantaneous dividend at time $t=0$ to reduce $\eta$ as far as possible, until the borrowing constraint binds so $\eta=\bar{\eta}$. The first inequality on $\theta$ in the proposition ensures that the borrowing constraint does indeed bind at a level of borrowing at which Equation (A6) has real roots.

The remaining inequality conditions on $\theta$ ensure that it is possible to achieve positive dividends per unit of capital (these are relatively weak conditions since normally $a>\delta$ in which case a policy of zero growth $g=0$ and no indebtedness will always yield positive dividends; but if depreciation is larger than the productivity of capital then a further restriction on $\theta$ is required). To establish these further conditions note that expected dividends per unit of capital $a-\delta+r \bar{\eta}-(1+\bar{\eta}) g-\frac{1}{2} \theta g^{2}$ are maximised by choosing $g=-(1+\bar{\eta}) \theta^{-1}$ resulting in expected dividend payments of $\lambda=$ $a-\delta+r \bar{\eta}+\frac{1}{2} \theta^{-1}(1+\bar{\eta})^{2}$. This is always greater than zero if $a>\delta$; otherwise this requires that $\theta<(1+\bar{\eta})^{2} / 2(\delta-a-r \bar{\eta})$.

This proof also shows that the Modigliani-Miller [16] proposition on the irrelevance of capital structure to the value of the firm applies, in this case when negative dividends are allowed. It does so in the sense that any net worth in excess of the minimum level $\bar{\eta} k$ is immediately paid out to shareholders and the firm always operates with maximum leverage. The value function is additive in $\bar{\eta} k$.

Finally, note that the fundamental valuation of a firm's capital by outside investors can be obtained by substituting $\rho=r, a=\bar{a}$ and $\bar{\eta}=0$ into this solution. A finite positive valuation is obtained provided the parameters satisfy:

$$
2 \frac{\bar{a}-\delta-r}{r^{2}}<\theta< \begin{cases}\infty & \text { if } \bar{a} \geq \delta \\ \frac{1}{2} \frac{1}{\delta-\bar{a}} & \text { if } \bar{a}<\delta\end{cases}
$$

in which case the growth rate (when held by outside investors) is given by

$$
\bar{g}=r-\sqrt{r^{2}-2 \theta^{-1}[\bar{a}-\delta-r]}
$$

and the value of the maximised objective by

$$
V=\frac{\bar{a}-\bar{g}-\delta-\frac{1}{2} \theta \bar{g}^{2}}{r-\bar{g}} k=(1+\theta \bar{g}) k .
$$

This provides an immediate proof of Proposition 1 in Section 3. 
Proof of Proposition 1. This valuation of the firm's assets by outside investors is also the maximum amount of debt that it can borrow from these investors, implying that the lower boundary for $\eta$ is given by Equation (3).

\section{Appendix B. Proofs of Propositions in Sections 3 and 4}

Proof of Proposition 5. (Proposition 3 requires no separate proof, since it is the special case when $\sigma_{2}=0$ and $\psi=1$ ). While uniqueness of solution can be established using standard arguments based on the non-convexity of the optimisation program, the proof provided here is geometric proof, offering some additional insights into both the existence of solution and its numerical calculation.

Applying standard methods of stochastic dynamic programming, with two state variables $k$ and $c$, the optimal policy by firms, at times when there is no recapitalisation $\left(\epsilon_{t}=0\right)$, satisfies the Hamilton-Jacobi-Bellman equation:

$$
\begin{aligned}
\rho V=\max _{i, \lambda, \psi}\left\{\lambda+\left[-\lambda+(\bar{a}+(a-\bar{a}) \psi) k+r c-i k-\frac{1}{2} \theta(i-\delta)^{2} k\right] V_{c}\right. \\
\left.+(i-\delta) k V_{k}+\frac{1}{2} \sigma_{1}^{2} \psi^{2} k^{2} V_{c c}+\frac{1}{2} \sigma_{2}^{2} \psi^{2} k^{2} V_{k k}\right\}
\end{aligned}
$$

with three first-order conditions for maximisation. The first is:

$$
\left\{\begin{array}{cc}
\lambda \geq 0 \text { of unbounded magnitude, } & V_{c}=1 \\
\lambda=0, & V_{c}>1
\end{array},\right.
$$

there is "bang-bang" control with two distinct regions of dividend behaviour: one when $c \geq c^{*}(k)$ with $V_{c}=1$ in which case the policy is to payout a discrete dividend to reduce cash holdings immediately to the dividend paying boundary $c^{*}$; the other when $c<c^{*}(k)$ wherein there is no payment of dividends and $V_{c}>1$. The second first-order condition is:

$$
(1+\theta(i-\delta)) V_{c}=V_{k}
$$

yielding the investment rule:

$$
i=\delta+\theta^{-1}\left(\frac{V_{k}}{V_{c}}-1\right) .
$$

The third first-order condition for maximisation (subject to the constraint $0 \leq \psi \leq 1$ ) is:

$$
(a-\bar{a}) k V_{c}+\psi k^{2}\left(\sigma_{1}^{2} V_{c c}+\sigma_{2}^{2} V_{k k}\right)=0
$$

yielding the final control rule:

$$
\psi=\max \left\{\min \left\{(a-\bar{a})\left[-k \frac{\sigma_{1}^{2} V_{c c}+\sigma_{2}^{2} V_{k k}}{V_{c}}\right]^{-1}, 1\right\}, 0\right\} .
$$

Due to the linearity of production the value function is linearly homogeneous in $k$ and so value can be expressed as a function $W$ of a single state variable $\eta=c / k$ :

$$
W(\eta)=k^{-1} V(c, k)=V(\eta, 1)
$$

implying the substitutions $V=k W, V_{c}=W^{\prime}, V_{k}=W-\eta W^{\prime}, q=V_{k} / V_{c}=W / W^{\prime}-\eta, V_{c c}=k^{-1} W^{\prime \prime}$, $V_{c k}=-k^{-1} \eta W^{\prime \prime}$ and $V_{k k}=k^{-1} \eta^{2} W^{\prime \prime}$ Substituting for both optimal policy and for $V$ and its derivatives yields Equation (14). The maximisation in this second boundary condition reflects the choice available to the firm when $\eta$ falls to $\bar{\eta}$; it may choose either to liquidate, in which case $W(\bar{\eta})=0$, or to recapitalise, which is worth doing if it can achieve a higher valuation by paying the fixed cost of recapitalisation $\chi k$ and increasing $\eta$ to $\eta^{*}$. The firm will never choose to recapitalise to a value of $\eta<\eta^{*}$. This is because when $\eta<\eta^{*}, V_{c}=W^{\prime}>1$, so the maximum possible value of $W(\bar{\eta})$ in Equation (7) is achieved by a full recapitalisation up to $\eta^{*}$. Turning to the uniqueness of this solution, note that as discussed in Appendix C solution of the upper boundary $\eta^{*}$ is characterised by Equation (10) (itself obtained from 
Equation (6) using $q=W / W^{\prime}-\eta$ which yields $\left.q^{\prime}=-W W^{\prime \prime} /\left(W^{\prime}\right)^{2}\right)$. Equation (10) can be written (allowing for dependencies of $\sigma$ on $\eta$ ) as:

$$
q^{\prime}=\frac{2}{\sigma_{1}^{2}+\eta^{2} \sigma_{2}^{2}} Q(q, \eta)(q+\eta)
$$

from which, since $\sigma_{1}^{2}+\eta^{2} \sigma_{2}^{2}>0$ and $q^{*}+\eta^{*}>\bar{q}+\bar{\eta} \geq 0$ and $Q(q, \eta)=a-\delta-(\rho-r) \eta-\rho q+$ $\frac{1}{2} \theta^{-1}(q-1)^{2}$ is a quadratic function of $q$ and linear function of $\eta$. This in turn implies that the possible locations of $q^{*}$ are given by $Q(q, \eta)=0$, i.e., a parabola in $(q, \eta)$ space, which when solved yields the location of $\eta$ on the dividend paying boundaries as a function of $q^{*}$ :

$$
\eta^{*}=\frac{a-\delta-\rho q^{*}+\frac{1}{2} \theta^{-1}\left(q^{*}-1\right)^{2}}{\rho-r}
$$

Inverting this equation to solve for $q=q^{*}$ on the dividend paying boundary yields:

$$
q^{*}=1+\theta\left(\rho \pm \sqrt{\rho^{2}-2 \theta^{-1}\left\{a-\delta-\rho-\eta^{*}(\rho-r)\right\}}\right)
$$

The uniqueness of the solution then follows (assuming continuity of $q(\eta)$ ) from noting that the value of $q=q^{*}$ is a function of the value of $q(\bar{\eta})=\bar{q}$ on the lower boundary. Given any starting value $\bar{q}$ the ODE characterising the solution can be computed (with $q^{\prime}>0$ ) until it meets $Q(q, \eta)=0$. There can only be one such intersection. Having crossed $Q(q, \eta)=0, q^{\prime}<0$ until there is another intersection, and this means any potential second intersection can only take place on the lower branch of $Q(q, \eta)=0$. However, in order for there to be an intersection on this lower branch it is necessary that the $q$-curve falls faster than the lower branch, i.e., that on the point of intersection:

$$
q^{\prime}<\left.\frac{\partial q}{\partial \eta}\right|_{Q(q, \eta)=0}<0
$$

which contradicts the requirement that $q^{\prime}=0$ on $Q(q, \eta)=0$. This contradiction shows that any solution of the ODE has at most one unique intersection with $Q(q, \eta)=0$.

This proof does not establish existence. While there can only be one solution to the ODE for $W$ satisfying the boundary conditions of Proposition 5, the existence of this solution is dependent on parameter values. Proposition 2 gave sufficient conditions for a solution to exist.

Proof of Proposition 2. First note that Equation (4a) is equivalent to

$$
\bar{\eta}>\eta_{\min }^{*}=-\frac{\rho-(a-\delta)+\frac{1}{2} \theta \rho^{2}}{\rho-r},
$$

where $\eta_{\min }^{*}$ is the minimum value of $\eta$ on the dividend paying boundary. This condition can be described as the "no-Ponzi" condition because, as stated in Proposition A1 in Appendix A when $\bar{\eta}>\eta_{\min }^{*}$ is satisfied, then the solution to the problem in the deterministic $\operatorname{limit}_{\lim } \lim _{\sigma \downarrow}$ exists, in which the growth of the fixed capital stock is less than the discount rate of firm shareholders $g<\rho$, and the value to shareholders comes from both growth of the capital stock and dividend payments.

The idea of proof is illustrated in Figure A1. Consider possible solutions of the ODE for $q(\eta)$. In the case of no recapitalisation, $\bar{q}=-\bar{\eta}$ and the lower intersection of $Q(q, \eta)=0$ with $\eta=\bar{\eta}$ is at $q=q_{-}^{*}=1+\theta\left(\rho-\sqrt{\rho^{2}-2 \theta^{-1}\{a-\delta-\rho-\bar{\eta}(\rho-r)\}}\right)$. This implies (using Equation (3)) that:

$$
q_{-}^{*}-\bar{q}=1+\theta\left(\rho-\sqrt{\rho^{2}-2 \theta^{-1}\{a-\delta-\rho-\bar{\eta}(\rho-r)\}}\right)+\bar{\eta}>0
$$

This shows that a solution with no recapitalisation exists, because the ODE begins at a point strictly below $q_{-}^{*}$, and since $q^{\prime}>0$ must eventually intersect with $Q(q, \eta)=0$. This in turn implies the existence of solutions with recapitalisation, since these are associated with higher values of $\bar{q}$ satisfying 
$-\bar{\eta}<\bar{q}<q_{-}^{*}$, in all cases with the ODE eventually intersecting with the lower branch of $Q(q, \eta)=0$; and with values of $\chi>0$. Eventually in the $\operatorname{limit} \lim _{\chi \downarrow 0} \bar{q}=q_{-}^{*}$.
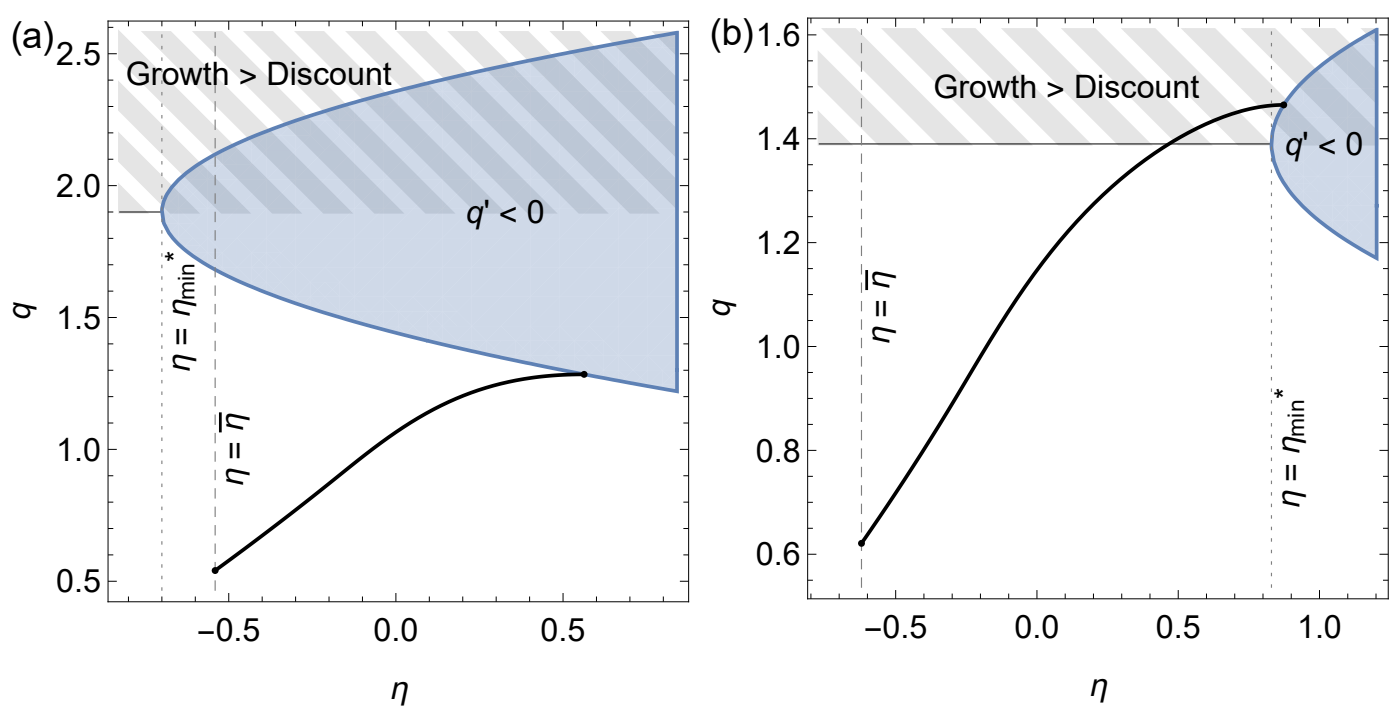

Figure A1. Illustration of Proof of Proposition 2. In subfigure (a), the "no-Ponzi" condition, Equation (A15), holds: the initial $\eta$ is below the dividend paying boundary, and so the solution is guaranteed to hit it. Subfigure (b) shows a scenario wherein the condition does not hold: The solution starts from a point to the left of $\eta_{\mathrm{min}}^{*}$ and grows fast enough to miss the lower branch of $q^{\prime}=0$ curve, entering a region where growth exceeds the discount rate. In (a), parameters are set to baseline, $\rho=0.06, r=0.05, \sigma_{1}=0.2, \sigma_{2}=0.0, a=0.1, \bar{a}=0.04, \delta=0.02, \theta=15$ and $\chi=0.75$; in (b), parameters are the same, except that $\theta=6.5$.

Some additional intuition into the factors that determine whether the "no-Ponzi" condition is satisfied or not can be obtained by re-expressing Equation (A15) as

$$
\bar{g}^{*}<\left[\frac{1}{2}\left(\rho-g^{*}\right)^{2} /(\rho-r)-\theta^{-1}\right]
$$

where

$$
\bar{g}^{*}=\left(r-\sqrt{r^{2}-2 \theta^{-1}[\bar{a}-\delta-r]}\right)<r
$$

is the rate of growth when capital stock is owned by external investors and

$$
g^{*}=\left(\rho-\sqrt{\rho^{2}-2 \theta^{-1}[\bar{a}-\delta-\rho]}\right)<\rho
$$

the rate of growth of the capital stock in the situation where firms can costlessly issued equity $(\chi=0)$ but are unable to borrow (see Appendix A). This expression indicates that in order for the "no-Ponzi" to be satisfied requires that the difference between the discount rates of firms and outside investors $\rho-r$ is comparatively small, or the net productivity of capital either in the hands of firms or investors $(a-\delta, \bar{a}-\delta)$ relative to the maximum values given by the constraints of Equations (4a) and (4b) is comparatively small, or the costs of adjustment of capital $\theta$ are comparatively high.

What about solution in the stochastic case if the "no-Ponzi" condition is not satisfied? A numerical solution can still be obtained and indicate that an optimal policy for choice of $\left\{i_{t}\right\},\left\{\lambda_{t}\right\},\left\{\epsilon_{\tau}\right\}$ satisfying the conditions of Proposition 3, i.e., with future dividend payments after any initial dividend payment to reduce $\eta$ to the desired target level $\eta^{*}$, may still exist, provided that $g^{*}$ is not too close to $\rho$.

Finally there are the propositions about the ergodic density. 
Proof of Proposition 4. (Proposition of Section 3 can again be obtained by imposing appropriate parameter restrictions.) Denote the density function for the location of firms across the possible values of $\eta$ at the moment $t$ by $f(t, \eta)$, with the corresponding cumulative density:

$$
F(t, \eta)=\int_{\bar{\eta}}^{\eta} f\left(t, \eta^{\prime}\right) \mathrm{d} \eta^{\prime}, \quad F\left(t, \eta^{*}\right)=1
$$

The evolution of $f(t, \eta)$ is then determined by the Kolmogorov forward or Fokker-Planck equation:

$$
\frac{\partial f}{\partial t}(t, \eta)=-\frac{\partial}{\partial \eta}\left[\mu^{\eta}(\eta) f(t, \eta)\right]+\frac{1}{2} \frac{\partial^{2}}{\partial \eta^{2}}\left[\sigma^{\eta}(\eta)^{2} f(t, \eta)\right],
$$

where $\eta$ follows the equation of motion

$$
\mathrm{d} \eta=\mu^{\eta} \mathrm{d} t+\sigma^{\eta} \mathrm{d} z
$$

with coefficients obtained simply by Itô differentiating $\eta=c / k$ :

$$
\mathrm{d} \eta=\frac{1}{k}\left[\mu^{c}-\eta \mu^{k}+\frac{\eta}{k}\left(\sigma^{k}\right)^{2}\right] \mathrm{d} t+\frac{\sigma^{c}}{k} \mathrm{~d} z_{1}-\eta \frac{\sigma^{k}}{k} \mathrm{~d} z_{2}
$$

where $\mu^{c, k}$ and $\sigma^{c, k}$ are respectively the drift and diffusion terms for $c$ and $k: \mathrm{d} c=\mu^{c} \mathrm{~d} t+\sigma^{c} \mathrm{~d} z_{1}$, $\mathrm{d} k=\mu^{k} \mathrm{~d} t+\sigma^{k} \mathrm{~d} z_{2}$; i.e.,

$$
\begin{gathered}
\mu^{c}=\left[\psi a+(1-\psi) \bar{a}-\delta+r \eta-\theta^{-1}(q-1)-\frac{1}{2} \theta^{-1}(q-1)^{2}\right] k, \\
\mu^{k}=\theta^{-1}(q-1) k, \\
\sigma^{c}=\sigma_{1} \psi k, \quad \sigma^{k}=\sigma_{2} \psi k .
\end{gathered}
$$

The increments $\mathrm{d} z_{1}, \mathrm{~d} z_{2}$ are independent and normally distributed, and so the noise sources in $\mathrm{d} \eta$ can be combined into a single term,

$$
k^{-1} \sigma^{c} \mathrm{~d} z_{1}-k^{-1} \eta \sigma^{k} \mathrm{~d} z_{2}=k^{-1} \sqrt{\left(\sigma^{c}\right)^{2}+\eta^{2}\left(\sigma^{k}\right)^{2}} \mathrm{~d} z .
$$

Substituting in the expressions for $\mu^{c, k}$ and $\sigma^{c, k}$ yields

$$
\begin{aligned}
\mathrm{d} \eta=\left[\bar{a}+(a-\bar{a}) \psi-\delta+r \eta-(1+\eta) \theta^{-1}\right. & (q-1) \\
& \left.-\frac{1}{2} \theta^{-1}(q-1)^{2}+\eta \sigma_{2}^{2} \psi^{2}\right] \mathrm{d} t+\sqrt{\sigma_{1}^{2}+\eta^{2} \sigma_{2}^{2}} \psi \mathrm{d} z .
\end{aligned}
$$

The ergodic probability density is then the stationary, $\partial f / \partial t=0$, solution of Equation (A16), also denoted by $f(\eta)$. Integration of the Kolmogorov forward equation in $\eta$ yields

$$
d=\mu^{\eta}(\eta) f(\eta)-\frac{1}{2} \frac{\partial}{\partial \eta}\left[\sigma^{\eta}(\eta)^{2} f(\eta)\right]
$$

It is convenient to write this in terms of $\phi$,

$$
\phi=\frac{\left(\sigma^{\eta}\right)^{2}}{2} f
$$

so this becomes:

$$
d=\left[\frac{\left(\sigma^{\eta}\right)^{2}}{2}\right]^{-1} \mu^{\eta}(\eta) \phi(\eta)-\frac{1}{2} \frac{\partial}{\partial \eta} \phi(\eta)
$$

and this yields Equation (15). 


\section{Appendix C. Numerical Solution}

\section{Appendix C.1. Preliminary Considerations and Some Economic Intuition}

The ordinary differential equation governing $q$ (Equation (17) for $\eta \leq \tilde{\eta}$ and Equation (10) for $\eta \geq \tilde{\eta}$ ) can be solved by forward integration using standard methods starting from any given initial condition $q(\bar{\eta})=\bar{q}$. The solution is completed by finding an intersection with $Q(q, \eta)=0$ on which $q^{\prime}=0$, if one exists, or establishing that there is no such intersection (moreover, any solution with an intersection with $Q(q, \eta)=0)$. Any initial value $\bar{q} \geq 1+\theta \rho$ can be ruled out, since it implies that $g>\rho$ for all $\eta$.

The inequality in Equation (A15), which is required if the special case of the model with no uncertainty $(\sigma=0)$ is to a be one with no "Ponzi-borrowing" and also ensures the existence of solution, leads to extremely straightforward numerical solution, since intersection with the lower branch of $Q(q, \eta)=0$ is guaranteed.

If however this inequality is not satisfied then for some values of $\bar{q}$, a value of $q^{*}$ whereat the ODE interacts with $Q(q, \eta)=0$ may be located on the upper boundary (if the ODE "misses" the lower branch, in which case it may or may not hit the upper branch). This considerably complicates the search for numerical solution because it is no longer possible to restrict the initial values $\bar{q}$ to a range of values for which intersection with $Q(q, \eta)=0$ is guaranteed.

Such solutions with upper branch intersections are of less economic interest than those where intersection is on the lower branch. It is possible that investment close to $\eta^{*}$ is so high that the firm has negative cash flow. This can be seen by substituting Equation (A13), $\psi=1, q=q^{*}$ and $\eta=\eta^{*}$ into Equation (A18), yielding the following expression for cash flow on the dividend paying boundary:

$$
\begin{aligned}
\mu^{\eta}=\frac{(a-\delta) \rho-\rho r+\left[(r-(a-\delta)) \theta^{-1}-\rho r\right]\left(q^{*}-1\right)}{\rho-r}+\frac{\frac{1}{2}(2 r+\rho) \theta^{-1}-\frac{1}{2} \theta^{-2}\left(q^{*}-1\right)}{\rho-r}\left(q^{*}-1\right)^{2}+\eta^{*} \sigma_{2}^{2}
\end{aligned}
$$

which, for sufficiently high $q^{*}$, is negative. The economic intuition in this case is similar to that applicable to the "Ponzi" solution of the model with no non-negativity constraint on dividends in Appendix A. The firm creates the most value not by dividend payments but from growing the capital stock at a rate close to and often above the shareholder's rate of discount, and this very high rate of investment can generate the negative expected cash flows.

The function $W\left(\eta^{*}\right)$ is obtained by substitution into Equation (A14) on the boundary $\eta^{*}$, using the boundary conditions $W^{\prime}=1$, to yield:

$$
W^{*}=\left(q^{*}+\eta^{*}\right) W^{\prime}=1+\eta^{*}+\rho \theta \pm \sqrt{2 \theta\left\{\left(\eta^{*}-\eta_{\text {min }}^{*}\right)(\rho-r)\right\}}
$$

with the positive root applying on the upper branch of $q^{*}$ and the negative root on the lower branch.

This in turn results in some useful insights into the solution. In the case of recapitalisation, Equation (7) can be written:

$$
0<W^{*}-\left(\eta^{*}-\bar{\eta}+\chi\right)=1+\rho \theta+\bar{\eta}-\chi \pm \sqrt{2 \theta\left\{\left(\eta^{*}-\eta_{\min }^{*}\right)(\rho-r)\right\}}<1+\rho \theta+\bar{\eta}
$$

where the first inequality is required by the maximisation in Equation (7) and the second because the presence of financing constraints must lower value $W(\bar{\eta})$ relative to the valuation for the case of no non-negativity constraint on dividend payments given by Equation (A3)). This establishes the following further proposition: 
Proposition A2. A solution with recapitalisation (for some sufficiently low value of $\chi$ ) exists. Let $\chi=\chi_{0}$ be the critical value of $\chi$ at which the firm is indifferent between recapitalisation and liquidation. Then: (i) If $\eta_{\text {min }}^{*} \leq \bar{\eta}$ a solution exists with $\eta^{*}$ on the lower branch of $Q(q, \eta)=0, \bar{q}$ satisfies:

$$
-\bar{\eta} \leq \bar{q}<\bar{q} \max =1+\theta\left(\rho-\sqrt{\rho^{2}-2 \theta^{-1}\{a-\delta-\rho-\bar{\eta}(\rho-r)\}}\right)>\bar{q}
$$

and the maximum possible value of $\eta^{*}$ satisfies:

$$
\eta_{\text {min }}^{*} \leq \eta^{*}<\eta_{\text {min }}^{*}+\frac{\left((\rho-r)+\sqrt{r^{2}-2 \theta^{-1}[\bar{a}-\delta-r]}-\theta^{-1} \chi_{0}\right)^{2}}{2(\rho-r)}
$$

(ii) If instead $\eta_{\text {min }}^{*}>\bar{\eta}$ then $-\bar{\eta} \leq \bar{q}<1+\rho \theta$; a solution may or may not exist solution may be on the upper branch of $Q(q, \eta)=0$, in which case $\eta^{*}$ satisfies:

$$
\eta_{\text {min }}^{*}<\eta^{*} \leq \eta_{\text {min }}^{*}+\frac{\chi^{2}}{2 \theta(\rho-r)}
$$

Proof. The existence of a solution with recapitalisation is guaranteed because $1+\rho \theta+\bar{\eta}=(\rho-r) \theta+$ $\theta \sqrt{r^{2}-2 \theta^{-1}[\bar{a}-\delta-r]}>0$. As noted above, solutions for which $\bar{q}>1+\theta \rho$ can be ruled out, and hence all possible solutions, with recapitalisation or without, are with an intersection of the ODE for $q$ on the lower branch of $Q(q, \eta)=0$; and (the value that applies when cost of recapitalisation $\chi=0$ and hence the maximum possible value of $\bar{q}$ ) is given by the intersection of this lower branch of Equation (A14) with $\eta=\bar{\eta}$. If solution is on the lower branch then the largest possible value of $\eta^{*}$ and the smallest value of $\bar{q}(\bar{q}=-\bar{\eta})$ arise when $\chi=\chi_{0}$ (the same solution also applies if $\chi$ is higher than this critical value and no-recapitalisation takes place). If $\chi=\chi_{0}$ then the first inequality in Equation (A23) binds and this implies the second inequality in Equation (A25). If instead solution is on the upper branch, then the largest possible value is when $\chi<\chi_{0}$, so that Equation (A23) binds and this implies the second inequality in Equation (A26).

Proposition A2 helps guide the numerical solution. If $\eta_{\min }^{*} \leq \bar{\eta}$ then a solution with a bounded value function exists and an intersection is guaranteed on the lower branch of $Q(q, \eta)=0$. A first calculation of the case with no recapitalisation determines $\chi_{0}$ and this can then be used to limit the scope of iteration on $\bar{q}$ (using Equation (A24)) in the search for solution in the case of recapitalisation. If instead $\eta_{\min }^{*}>\bar{\eta}$ then a solution with a bounded value function and finite $\eta^{*}$ may not exist. The existence of a solution for the case of no-recapitalisation can be established by computing the ODE Equation (10) upwards. If $\eta$ exceeds the upper bound given by Equation (A26) then there is no intersection and no solution) is not satisfied, then while there is an intersection there is no finite solution to the value function. A solution with recapitalisation will exist for at least some values of $\chi$ if there is a solution for no-recapitalisation. The proposition then provides a slightly different limits on the scope of iteration on $\bar{q}$ and the same criteria can be applied to establish if there is an intersection with $Q(q, \eta)=0$, and if so whether this represents a finite value for the value function.

\section{Appendix C.2. Model without Option to Rent}

For any given $\bar{q}$, the right-hand side boundary at $\eta=\eta^{*}$ wherein $q^{\prime}\left(\eta^{*}\right)=0$ is found by evaluating the function $q^{\prime}(\eta)$ during the integration. After a single integration step is found to bracket a root of $q^{\prime}(\eta)$, the critical value of $\eta$ is pin-pointed using standard root finding methods, here the Brent's method.

The value function $W$ can be solved from $W^{\prime}=W /(\eta+q)$ parallel to integrating the equation for $q$. The boundary condition $W^{\prime \prime}\left(\eta^{*}\right)=0$ will be satisfied since the $q$ variable integration is stopped at $q^{\prime}=0$. In order to also satisfy the boundary condition $W^{\prime}\left(\eta^{*}\right)=1$, solve $W$ for an arbitrary initial value at $\bar{\eta}$. Let the resulting solution be $\tilde{W}$. Since the ODE for $W$ is linear and homogeneous, simply multiply $\tilde{W}$ ex post by $\left[\tilde{W}^{\prime}\left(\eta^{*}\right)\right]^{-1}$ to get a solution for which $W^{\prime}\left(\eta^{*}\right)=1$. 
In the case of liquidation, the lower boundary is $\bar{\eta}=-\bar{q}$, and consequently, the derivative of $W, W^{\prime}=W /(\eta+q)$ cannot be evaluated. Appendix $\mathrm{D}$ shows that $W \propto \eta-\bar{\eta}$, and so $W^{\prime}(\bar{\eta})$ is finite. Thus, if $\bar{\eta}$ is indeed liquidating, we simply set $\tilde{W}^{\prime}(\bar{\eta})=1$ and $W(\bar{\eta})=0$.

Solving for the ergodic density with an absorbing boundary requires determining the constant of integration (the rate of flow across the boundary) $d$ and this requires two boundary conditions. These conditions are that the absorbing boundary must have a zero density, i.e., $f(\bar{\eta})=0$, and the cumulative density must satisfy $F\left(\eta^{*}\right)=1$.

The following method enforces these conditions. Solve two independent differential equations for two densities $f_{0}$ and $f_{1}$ satisfying:

$$
f_{0}^{\prime}(\eta)=\frac{2 \mu(\eta)}{\sigma^{2}} f_{0}(\eta), \quad f_{1}^{\prime}(\eta)=\frac{2 \mu(\eta)}{\sigma^{2}} f_{1}(\eta)+1
$$

These are obtained by integration starting from arbitrary non-zero initial conditions. Let $F_{0}$ and $F_{1}$ be the resulting corresponding cumulative functions, with $F_{0}^{\prime}=f_{0}, F_{1}^{\prime}=f_{1}$ and $F_{0}(\bar{\eta})=F_{1}(\bar{\eta})=0$. This determines values for $F\left(\eta^{*}\right)$ and $F_{1}\left(\eta^{*}\right)$.

Then find the ergodic density by choosing appropriate constants $a_{0}$ and $a_{1}$ in the following function $f$ :

$$
f(\eta)=a_{0} f_{0}(\eta)+a_{1} f_{1}(\eta),
$$

These coefficients $a_{0}, a_{1}$ are determined by the conditions $f(\bar{\eta})=0$ and $F\left(\eta^{*}\right)=1$ as follows. Upon substituting the trial solution (A28), one obtains

$$
a_{0} f_{0}(\bar{\eta})+a_{1} f_{1}(\bar{\eta})=0, \quad a_{0} F_{0}\left(\eta^{*}\right)+a_{1} F_{1}\left(\eta^{*}\right)=1 .
$$

yielding a pair of linear equations that can be solved for $a_{0}$ and $a_{1}$. To obtain $d$ differentiate (A28) and use Equation (A27), to get:

$$
f^{\prime}(\eta)=\frac{2 \mu}{\sigma^{2}} f(\eta)+a_{1}
$$

so $a_{1}=-2 d / \sigma^{2}$ (cf. Equation (A19)).

The possibility for recapitalisation is tested by finding roots of

$$
G(\bar{q})=W\left[\bar{q}, \eta^{*}(\bar{q})\right]-W(\bar{q}, \bar{\eta})-\left[\eta^{*}(\bar{q})-\bar{\eta}\right]-\chi,
$$

making explicit the dependence of the location of the upper dividend paying boundary $\eta=\eta^{*}$ and the function $W(\eta)$ on the value of $q$ on the lower boundary $q(\bar{\eta})=\bar{q}$. Clearly $G=0$ is equivalent to achieving Equation (7). Functions $\eta^{*}, q$ and $W$ are all obtained using the same method outlined above (i.e., jointly computing the two odes for $q$ and $W$ using $\bar{q}$ and an arbitrary value of $W$ on $\bar{\eta}$, locating $\eta^{*}$ from $q^{\prime}=0$, and rescaling $W$ to enforce $W^{\prime}=1$ ).

The task then is to iterate on the starting value $\bar{q}$ to find the root of $G(\bar{q})$. First a coarse root bracketing is attempted by evaluating $G$ at $\bar{q}_{i}=-\bar{\eta}+\left(q_{1}+\bar{\eta}\right) i / n_{q}$, where $i=0 \ldots n_{q}$ and $n_{q}$ an integer (using $n_{q}=10$ ), and $q_{1}$ is $q$ as given by Equation (A14) if that value is real, or $1+\theta \rho$ if it is not. If sign of $G$ changes across a bracketing interval $\left(\bar{q}_{i}, \bar{q}_{i+1}\right)$, the root is pin-pointed using standard root finding algorithms. This locates a recapitalisation solution. If no roots are found, or a root is found with $\bar{q}<-\bar{\eta}$ or $q^{*}>1+\theta \rho$ then the solution is identified as liquidation with $\bar{q}=-\bar{\eta}$.

\section{Appendix C.3. Model with Option to Rent}

The algorithm outline is same as in the model without the rental option. However, the solution near the lower boundary is more involved when recapitalisation is not undertaken, and so $\psi(\bar{\eta})=0$.

The differential equations for $q$ can again be solved by simple forward integration starting from $q(\bar{\eta})=\bar{q}$. If recapitalisation is available $(\bar{q}>-\bar{\eta})$, no singularities are present, and the equation for $q$, Equation (17), can be integrated directly to obtain $q(\eta), \eta^{*}$ and now also $\tilde{\eta}$. The point $\tilde{\eta}$ is found in the 
same way as $\eta^{*}$, i.e., by monitoring the function $\psi-1$ as integration advances and polishing the root after a coarse approximation is found. Initial $\bar{q}$ is found the same way as for the model without renting (but with $q, W$ computed slightly differently as described below).

If $\bar{q}=-\bar{\eta}$, then $\psi=0$ and singularities appear. As is shown in Appendix D, the derivative $q^{\prime}(\bar{\eta})$ is finite. In order to evaluate it numerically, use Equation (A32) since Equation (17) is indeterminate at $\bar{\eta}$ (in practice, numerical round-off would cause significant error in $\bar{q}$ ). Otherwise the solution of $q$ proceeds the same way as with a recapitalising lower boundary.

Using $\phi$, and expanding the resulting equation in the renting $(0<\psi<1)$ and not renting regimes $(\psi=1)$, yields

$$
\phi^{\prime}=\left\{\begin{array}{l}
\frac{\bar{a}+(a-\bar{a}) \psi-\delta+r \eta+\sigma_{2}^{2} \psi^{2} \eta-\theta^{-1}(q-1)\left[\eta+\frac{1}{2}(q+1)\right]}{\frac{1}{2}\left(\sigma_{1}^{2}+\eta^{2} \sigma_{2}^{2}\right) \psi^{2}} \phi-d, \\
\text { when } \psi \in(0,1) \\
\frac{a-\delta+r \eta+\sigma_{2}^{2} \eta-\theta^{-1}(q-1)\left[\eta+\frac{1}{2}(q+1)\right]}{\frac{1}{2}\left(\sigma_{1}^{2}+\eta^{2} \sigma_{2}^{2}\right)} \phi-d \\
\text { when } \psi=1 .
\end{array}\right.
$$

When there are no recapitalisation, equations for $f^{\prime}$ and $W^{\prime}$, unlike that for $q^{\prime}$, do not tend to finite values at $\bar{\eta}$, since $\psi(\bar{\eta})=0$ if $q(\bar{\eta})=-\bar{\eta}, q^{\prime}(\bar{\eta})>0$. Due to this divergence, the point $\bar{\eta}$ cannot be reached by directly integrating the model equations, which in principle could be done backwards from, say, $\tilde{\eta}$ down to $\bar{\eta}+\epsilon, 0<\epsilon \ll 1$. Cutting the integration short in this way would lead to severe underestimation of the probability mass near $\bar{\eta}$ if $f$ diverges fast enough at this edge.

This issue is resolved using the analytically obtained power-law solutions, $f_{\mathrm{a}} \propto(\eta-\bar{\eta})^{\alpha-2}$ (Equation (21)) and $W_{\mathrm{a}} \propto(\eta-\bar{\eta})^{\beta}$ (Equation (18)), from $\bar{\eta}$ up to a cross-over value $\eta_{\times}$. Numerical solutions are matched to the analytic ones so that the resulting functions are continuous. The cross-over point can determined by requiring that

$$
\left|\frac{f_{\mathrm{a}}^{\prime}\left(\eta_{\times}\right)}{f_{\mathrm{a}}\left(\eta_{\times}\right)}\right|=\varepsilon^{-1}
$$

where $0<\epsilon \ll 1$, implying that the divergent terms dominate the expression for the derivative of $f$. However, since $W^{\prime}$ also tends to infinity, the same condition applies to $W_{\mathrm{a}}$ as well. This gives two different cross-over values; the smallest is chosen:

$$
\eta_{\times}=\varepsilon \min (|\alpha|, \beta)+\bar{\eta},
$$

where $\alpha$ is given by Equation (A34) and $\beta=1 /\left(1+q^{\prime}(\bar{\eta})\right)$, with $q^{\prime}(\bar{\eta})$ from Equation (A32). The results reported here use the value $\varepsilon=1.0 \times 10^{-3}$ and the analytic solution for $f$ to obtain the cumulative density $F$ below $\eta_{\times}$.

If the lower boundary is at $\bar{q}=-\bar{\eta}$, then directly integrate Equation (A29) with $d=0$ from $\eta_{\times}$to $\eta^{*}$. The obtained solution can then be multiplied by a constant to make the cumulative distribution satisfy $F\left(\eta^{*}\right)=1$. If $\bar{\eta}$ is absorbing (recapitalisation), use the same trick as in the model without rent: solve for $\phi_{0}$ and $\phi_{1}$ satisfy Equation (A29) with $d=0$ and $d=1$, respectively. The final $\phi$ is then constructed as a superposition of these two, $\phi=a_{0} \phi_{0}+a_{1} \phi_{1}$. Coefficients $a_{0}$ and $a_{1}$ are determined from

$$
\phi(\bar{\eta})=0, \quad \int_{\bar{\eta}}^{\eta^{*}} \frac{2}{\left(\sigma^{\eta}(\eta)\right)^{2}} \phi(\eta) \mathrm{d} \eta=1
$$

When needed, the same analytic solution, Equation (21), can be used for both $\phi_{0}$ and $\phi_{1}\left(\phi_{0,1} \propto\right.$ $\left.(\eta-\bar{\eta})^{\alpha} /\left(\sigma^{\eta}\right)^{2}\right)$, since $d$ term is negligible near $\bar{\eta}$. 
Note that reverting to the analytic solution for $f$ is equivalent to using a truncated integration range with an additional correction term coming from the analytical solution near $\bar{\eta}$. Numerical simulations confirm that this approach is sound: (i) the analytical and numerical solutions are in very good agreement across a wide range of $\eta$, (ii) the obtained solutions are independent of $\epsilon$, provided it is small enough while keeping the numerical solution from reaching the singularity, and (iii) qualitative features of the solution do not change if the analytical correction is omitted.

\section{Appendix D. Behaviour of Solutions Near Boundaries}

This Appendix provides the derivation of the asymptotic approximations summarised in Proposition 7.

\section{Appendix D.1. Model without Option to Rent}

While no singularities emerge in the model with no option to rent, it is still useful to begin with this simple case. The evolution of the value function $W$ is given by $W^{\prime} / W=1 /(q+\eta)$, which in the case of liquidation tends to infinity as the point of maximum borrowing where $q(\bar{\eta})=-\bar{\eta}$ is approached. This means there is a potential singularity in $W$ at $\bar{\eta}$. It can be shows that in the model without the option to rent this does not occur and $W$ is linear close to $\eta=\bar{\eta}$.

Suppose now that $q$ is of the form $q(\eta)=-\bar{\eta}+q^{\prime}(\bar{\eta})(\eta-\bar{\eta})+\mathcal{O}\left((\eta-\bar{\eta})^{2}\right)$. Near the boundary, $W$ follows

$$
W^{\prime}=\frac{1}{1+q^{\prime}(\bar{\eta})} \frac{W}{\eta-\bar{\eta}}+\mathcal{O}(\eta-\bar{\eta})
$$

The solution is then given by Equation (18) in the main text. In the case of the model without renting, it is clear from Equation (10) that $q^{\prime}(\bar{\eta})=0$ and so $W$ is linear near $\bar{\eta}$.

\section{Appendix D.2. Model with Option to Rent}

Turning to the model with option to rent, again a singularity can occur only on the lower boundary and only when there is no recapitalisation, i.e., when $q(\bar{\eta})=-\bar{\eta}$ and $\psi(\bar{\eta})=0$.

Note now that in the equation for $q^{\prime}$, Equation (17), both the numerator and the denominator vanish. Applying the l'Hopital's rule, the derivative can be solved as:

$$
q^{\prime}(\bar{\eta})=\frac{-(\rho-r-\gamma) \pm \sqrt{(\rho-r-\gamma)^{2}+4 \gamma \theta^{-1}[1+\theta \rho-\bar{q}]}}{2 \theta^{-1}[1+\theta \rho-\bar{q}]}
$$

where $\gamma=(a-\bar{a})^{2} / 2\left(\sigma_{1}^{2}+\bar{\eta}^{2} \sigma_{2}^{2}\right)$. Above, only the plus sign applies. This can be seen by recalling that $\bar{q}<q_{\max }=1+\rho \theta$ must apply (see above for the reasoning), in which case only the plus sign gives a positive $q^{\prime}$. Thus, the solution near $\bar{\eta}$ is given by Equation (19) in the main text.

The power-law form of $W$ given in Equation (18) holds here as well. Since now $q^{\prime}(\bar{\eta})>0$, the exponent $\beta=1 /\left(1+q^{\prime}(\bar{\eta})\right)$ is always less than one, in contrast to the model without option to rent, implying that $\lim _{\eta \downarrow \bar{\eta}} W^{\prime}=\lim _{\eta \downarrow \bar{\eta}}\left(-W^{\prime \prime} / W^{\prime}\right)=+\infty$.

To find the behaviour of the ergodic density near $\bar{\eta}, \bar{q}$, requires $\psi$. This time $\eta-\bar{\eta}$ is not negligible compared to $q-\bar{q}$. A straight-forward calculation gives:

$$
\psi=\psi^{\prime}(\bar{\eta})(\eta-\bar{\eta})
$$

where

$$
\psi^{\prime}(\bar{\eta})=\frac{2}{a-\bar{a}}\left\{\rho-r+\theta^{-1}[1+\theta \rho-\bar{q}] q^{\prime}(\bar{\eta})\right\} .
$$

Next, the $\eta \rightarrow \bar{\eta}$ limiting forms of $q$ and $\psi$ are substituted into Equation (A20), and only terms up to $\mathcal{O}(\eta-\bar{\eta})$ are kept. Notice that the numerator vanishes in the leading order, and hence $\phi^{\prime} \propto(\eta-\bar{\eta})^{-1}$ and not $\propto(\eta-\bar{\eta})^{-2}$ :

$$
\phi^{\prime}=\alpha \frac{\phi}{\eta-\bar{\eta}^{\prime}}
$$


where

$$
\alpha=\frac{(a-\bar{a}) \psi^{\prime}(\bar{\eta})+r-\frac{1}{2} \theta^{-1} q^{\prime}(\bar{\eta})(\bar{\eta}+1)+\theta^{-1}(\bar{\eta}+1)\left(1+q^{\prime}(\bar{\eta}) / 2\right)}{\frac{1}{2}\left(\sigma_{1}^{2}+\bar{\eta}^{2} \sigma_{2}^{2}\right) \psi^{\prime}(\bar{\eta})^{2}} .
$$

This gives the power-law solution Equation (20) in the main text. Finally using Equation (A33) yields Equation (21) of the main text.

\section{References}

1. Milne, A.; Robertson, D. Firm Behaviour Under the Threat of Liquidation. J. Econ. Dyn. Control 1996, 20, 1427-1449.

2. Brunnermeier, M.K.; Sannikov, Y. A Macroeconomic Model with a Financial Sector. Am. Econ. Rev. 2014, 104, 379-421, doi:10.1257/aer.104.2.379.

3. Whittle, P. Optimization over Time: Dynamic Programming and Stochastic Control; Wiley-Blackwell: New York, NY, USA, 1982; Volume 1.

4. Carroll, C.D. A theory of the consumption function, with and without liquidity constraints. J. Econ. Perspect. 2001, 15, 23-45.

5. Arrow, K.J.; Harris, T.; Marschak, J. Optimal inventory policy. Econometrica 1951, 19, $250-272$.

6. Scarf, H. The Optimality of (sS) Policies in the Dynamic Inventory Problem. Mathematical Methods in the Social Science; Arrow, K.J., Karlin, S., Suppes, P., Eds.; Stanford University Press: Stanford, CA, USA, 1960; Chapter 22

7. Jorgenson, D.W. Capital Theory and Investment Behavior. Am. Econ. Rev. 1963, 53, 247-259, doi:10.2307/1823868.

8. Lucas, R.E., Jr; Prescott, E.C. Investment under Uncertainty; Princeton University Press: Princeton, NJ, USA, 1971; Volume 39, pp. 659-681.

9. Miller, M.H.; Orr, D. A Model of the Demand for Money by Firms. Q. J. Econ. 1966, 80, 413-435, doi:10.2307/1880728.

10. Constantinides, G.M. Stochastic Cash Management with Fixed and Proportional Transaction Costs. Manag. Sci. 1976, 22, 1320-1331, doi:10.2307/2630145.

11. Frenkel, J.A.; Jovanovic, B. On Transactions and Precautionary Demand for Money. Q. J. Econ. 1980, 95, 25-43, doi:10.2307/1885347.

12. Merton, R.C. On the Pricing of Corporate Debt: The Risk Structure of Interest Rates. J. Financ. 1974, $29,449-470$.

13. Anderson, R.W.; Sundaresan, S. Design and valuation of debt contracts. Rev. Financ. Stud. 1996, 9, 37-68.

14. Mella-Barral, P.; Perraudin, W. Strategic debt service. J. Financ. 1997, 52, 531-556.

15. Leland, H.E. Agency Costs, Risk Management, and Capital Structure. J. Financ. 1998, 53, 1213-1243, doi:10.1111/0022-1082.00051.

16. Modigliani, F.; Miller, M.H. The Cost of Capital, Corporation Finance and the Theory of Investment. Am. Econ. Rev. 1958, 48, 261-297, doi:10.2307/1809766.

17. Tirole, J. The Theory of Corporate Finance; Princeton University Press: Princeton, NJ, USA, 2006.

18. Myers, S.C. The Capital Structure Puzzle. J. Financ. 1984, 39, 574-592, doi:10.1111/j.1540-6261.1984.tb03646.x.

19. Myers, S.C.; Majluf, N.S. Corporate financing and investment decisions when firms have information that investors do not have. J. Financ. Econ. 1984, 13, 187-221, doi:10.1016/0304-405X(84)90023-0.

20. Froot, K.; Scharfstein, D.; Stein, J.C. Risk Management: Coordinating Corporate Investment and Financing Policies. J. Financ. 1993, 48, 1629-1658, doi:10.1111/j.1540-6261.1993.tb05123.x.

21. Gertler, M. Financial Capacity and Output Fluctuations in an Economy with Multi-Period Financial Relationships. Rev. Econ. Stud. 1992, 59, 455, doi:10.2307/2297859.

22. Townsend, R.M. Optimal contracts and competitive markets with costly state verification. J. Econ. Theory $1979,21,265-293$.

23. Sannikov, Y. A Continuous-Time Version of the Principal-Agent Problem. Rev. Econ. Stud. 2008, 75, 957-984, doi:10.1111/j.1467-937X.2008.00486.x.

24. De Marzo, P.M.; Sannikov, Y. Optimal Security Design and Dynamic Capital Structure in a Continuous-Time Agency Model. J. Financ. 2006, 61, 2681-2724, doi:10.1111/j.1540-6261.2006.01002.x. 
25. Mauer, D.C.; Triantis, A.J. Interactions of corporate financing and investment decisions: A dynamic framework. J. Financ. 1994, 49, 1253-1277.

26. Radner, R.; Shepp, L. Risk vs. profit potential: A model for corporate strategy. J. Econ. Dyn. Control 1996, 20, 1373-1393, doi:10.1016/0165-1889(95)00904-3.

27. Jeanblanc-Picqué, M.; Shiryaev, A.N. Optimization of the flow of dividends. Russ. Math. Surv. 1995, 50, 257-277, doi:10.1070/RM1995v050n02ABEH002054.

28. Asmussen, S.; Taksar, M. Controlled diffusion models for optimal dividend pay-out. Insur. Math. Econ. 1997, 20, 1-15, doi:10.1016/S0167-6687(96)00017-0.

29. Taksar, M.I.; Zhou, X.Y. Optimal risk and dividend control for a company with a debt liability. Insur. Math. Econ. 1998, 22, 105-122, doi:10.1016/S0167-6687(98)00012-2.

30. Jgaard, B.H.; Taksar, M. Controlling risk exposure and dividends payout schemes: Insurance company example. Math. Financ. 1999, 9, 153-182.

31. Asmussen, S.; Højgaard, B.; Taksar, M. Optimal risk control and dividend distribution policies. Example of excess-of loss reinsurance for an insurance corporation. Financ. Stochastics 2000, 4, 299-324.

32. Milne, A.; Whalley, A.E. Bank Capital and Risk Taking; Bank of England Working Paper: London, UK, 1999.

33. Milne, A.; Whalley, A.E. Bank Capital Regulation and Incentives for Risk Taking; Cass Business School Research Paper: London, UK, 2002.

34. Milne, A. The Inventory Perspective on Bank Capital; Cass Business School Research Paper: London, UK, 2004.

35. Peura, S.; Keppo, J. Optimal Bank Capital With Costly Recapitalization. J. Bus. 2006, 79, 2163-2201, doi:10.2139/ssrn.685786.

36. Krugman, P.R. Target Zones and Exchange Rate Dynamics. Q. J. Econ. 1991, 106, 669-682, doi:10.2307/2937922.

37. Mundaca, G.; Øksendal, B. Optimal stochastic intervention control with application to the exchange rate. J. Math. Econ. 1998, 29, 225-243, doi:10.1016/S0304-4068(97)00013-X.

38. Korn, R. Some applications of impulse control in mathematical finance. Math. Methods Oper. Res. (ZOR) 1999, 50, 493-518, doi:10.1007/s001860050083.

39. Rochet, J.C.; Villeneuve, S. Liquidity management and corporate demand for hedging and insurance. J. Financ. Intermediation 2011, 20, 303-323, doi:10.1016/j.jfi.2010.11.001.

40. Bolton, P.; Chen, H.; Wang, N. A Unified Theory of Tobin's q, Corporate Investment, Financing, and Risk Management. J. Financ. 2011, 66, 1545-1578, doi:10.1111/j.1540-6261.2011.01681.x.

41. Bolton, P.; Chen, H.; Wang, N. Market timing, investment, and risk management. J. Financ. Econ. 2013, 109, 40-62, doi:10.1016/j.jfineco.2013.02.006.

42. Rampini, A.A.; Viswanathan, S. Collateral and capital structure. J. Financ. Econ. 2013, 109, 466-492, doi:10.1016/j.jfineco.2013.03.002.

43. Palazzo, B. Cash holdings, risk, and expected returns. J. Financ. Econ. 2012, 104, 162-185, doi:10.1016/j.jfineco.2011.12.009.

44. Anderson, R.W.; Carverhill, A. Corporate Liquidity and Capital Structure. Rev. Financ. Stud. 2011, 25, 797-837, doi:10.1093/rfs/hhr103.

45. Gamba, A.; Triantis, A. The Value of Financial Flexibility. J. Financ. 2008, 63, 2263-2296, doi:10.1111/j.1540-6261.2008.01397.x.

46. Gamba, A.; Triantis, A.J. Corporate Risk Management: Integrating Liquidity, Hedging, and Operating Policies. Manag. Sci. 2014, 60, 246-264, doi:10.1287/mnsc.2013.1752.

47. Greenwald, B.; Stiglitz, J.E.; Weiss, A. Informational imperfections in the capital market and macroeconomic fluctuations. Am. Econ. Rev. 1984, pp. 194-199.

48. Kiyotaki, N.; Moore, J. Credit Cycles. J. Political Econ. 1997, 105, 211-248.

49. Bernanke, B.S.; Gertler, M. Agency Costs, Net Worth, and Business Fluctuations. Am. Econ. Rev. 1989, 79, 14-31.

50. Bernanke, B.; Gertler, M.; Gilchrist, S. The Financial Accelerator in a Quantitative Business Cycle Framework. In Handbook of Macroeconomics, Volume 1C; Taylor, J.B.; Woodford, M., Eds.; Elsevier Science: North-Holland, The Netherlands, 1999; pp. 1341-1393.

51. Danielsson, J.; Shin, H.S.; Zigrand, J.P. The impact of risk regulation on price dynamics. J. Bank. Financ. 2004, $28,1069-1087$. 
52. Brunnermeier, M.K.; Pedersen, L.H. Market Liquidity and Funding Liquidity. Rev. Financ. Stud. 2008, 22, 2201-2238, doi:10.1093/rfs/hhn098.

53. Adrian, T.; Boyarchenko, N. Intermediary Leverage Cycles and Financial Stability; Federal Reserve Bank of New York Staff Reports No 567; Federal Reserve Bank of New York: New York, NY, USA, 2013.

54. Isohätälä, J.; Klimenko, N.; Milne, A. Post-Crisis Macrofinancial Modeling: Continuous Time Approaches; Palgrave Macmillan UK: London, UK, 2016, doi:10.1007/978-1-137-49449-8.

55. He, Z.; Krishnamurthy, A. A Model of Capital and Crises. Rev. Econ. Stud. 2012, 79, 735-777.

56. He, Z.; Krishnamurthy, A. Intermediary Asset Pricing. Am. Econ. Rev. 2013, 103, 732-770.

(C) 2020 by the authors. Licensee MDPI, Basel, Switzerland. This article is an open access article distributed under the terms and conditions of the Creative Commons Attribution (CC BY) license (http:/ / creativecommons.org/licenses/by/4.0/). 